\title{
REGULARITY RESULTS FOR FULLY NONLINEAR INTEGRO-DIFFERENTIAL OPERATORS WITH NONSYMMETRIC POSITIVE KERNELS : SUBCRITICAL CASE
}

\author{
YONG-CHEOL KIM AND KI-AHM LEE
}

\begin{abstract}
АвsтRAct. We introduce a new class of fully nonlinear integro-differential operators with possible nonsymmetric kernels. For the index $\sigma$ of the operator in $(1,2)$ (subcritical case), we introduce very general class of fully nonlinear integro-differential operators and obtain a comparison principle, a nonlocal version of the Alexandroff-Backelman-Pucci estimate, a Harnack inequality, a Hölder regularity, and an interior $C^{1, \alpha}$-regularity for equations associated with such a class.
\end{abstract}

\section{INTRODUCTION}

It is well-known from general theory on semigroups that the infinitesimal generator of any Lèvy process always exists for all functions in the Schwartz space $\mathfrak{S}\left(\mathbb{R}^{n}\right)$. From the celebrated Lèvy-Khintchine formula, we can derive that the infinitesimal generator is given by an operator of the general form

$$
\begin{aligned}
\mathcal{L} u(x)=\sum_{i=1}^{n} & \sum_{j=1}^{n} a_{i j} \partial_{i j} u+\sum_{i=1}^{n} b_{i} \partial_{i} u \\
& +\int_{\mathbb{R}^{n} \backslash\{0\}}\left[u(x+y)-u(x)-(\nabla u(x) \cdot y) \chi_{B_{1}}(y)\right] \mathrm{dm}(y) .
\end{aligned}
$$

The first term corresponds to the diffusion, the second to the drift, and the third to the jump part.

In this paper, we shall focus on the operators which are obtained in purely jump processes, i.e. processes without diffusion or drift part. In particular, we are mainly concerned with the operators with the general form

$$
\mathcal{L}^{t} u(x)=\int_{\mathbb{R}^{n} \backslash\{0\}}\left[u(x+y)-u(x)-(\nabla u(x) \cdot y) \chi_{B_{t}}(y)\right] \mathrm{d} \mathfrak{m}(y), t>0,
$$

where $m$ is a positive measure satisfying $\int_{\mathbb{R}^{n} \backslash\{0\}}\left(|y|^{2} \wedge 1\right) d \mathfrak{d}(y)<\infty$, where $|y|^{2} \wedge 1=\min \left\{|y|^{2}, 1\right\}$. The value of $\mathcal{L}^{t} u(x)$ is well-defined when $u$ is bounded on $\mathbb{R}^{n}$ and $C^{1,1}$ at $x$. These concepts shall be defined more precisely later. The operator $\mathcal{L}^{t}$ described above is called a linear integro-differential operator.

The operator (1.0.2) was introduced with too much generality. So we shall restrict our attention to the operators $\mathcal{L}^{t}$ where the measure $m$ is given

2000 Mathematics Subject Classification: 47G20, 45K05, 35J60, 35B65, 35D10 (60J75). 
by a positive kernel $K$ which is not necessarily symmetric. That is to say, the operators $\mathcal{L}^{t}$ are given by

$$
\mathcal{L}^{t} u(x)=\text { p.v. } \int_{\mathbb{R}^{n}}\left[u(x+y)-u(x)-(\nabla u(x) \cdot y) \chi_{B_{t}}(y)\right] K(y) d y, t>0 .
$$

Then we see that $\mathcal{L}^{t} u(x)$ is well-defined provided that $u \in C^{1,1}(x) \cap \mathrm{B}\left(\mathbb{R}^{n}\right)$ (refer to Definition 2.1.3 for the definition of $C^{1,1}(x)$ ) where $\mathrm{B}\left(\mathbb{R}^{n}\right)$ denotes the family of all real-valued bounded functions defined on $\mathbb{R}^{n}$.

If $K$ is symmetric, then an odd function $\left[(\nabla u(x) \cdot y) \chi_{B_{t}}(y)\right] K(y)$ will be canceled in the integral, and so we have that

$$
\mathcal{L}^{t} u(x)=\text { p.v. } \int_{\mathbb{R}^{n}}[u(x+y)+u(x-y)-2 u(x)] K(y) d y, t>0 .
$$

On the other hand, if $K$ is not symmetric, the effect of $\left[(\nabla u(x) \cdot y) \chi_{B_{t}}(y)\right] K(y)$ persists and we can actually observe that the influence of this gradient term becomes stronger as we try to get an estimate in smaller regions. The aim of this work is to obtain regularity results for fully nonlinear integrodifferential equations with possible nonsymmetric kernels.

This kind of equations are often obtained in stochastic control problems [S]. If a player in a stochastic game is permitted to choose different strategies at every step in order to maximize the expected value of some function at the first exit point of a domain, then a convex nonlinear operator

$$
\mathcal{I}^{t} u(x)=\sup _{\alpha} \mathcal{L}_{\alpha}^{t} u(x), t>0
$$

appears in the game. In a competitive game with two or more players, more complicated operators of the form

$$
\mathcal{I}^{t} u(x)=\inf _{\beta} \sup _{\alpha} \mathcal{L}_{\alpha \beta}^{t} u(x), t>0,
$$

can be obtained. The different aspect between (1.0.3) and 1.0.4) is convexity. Also an operator like $\mathcal{I}^{t} u(x)=\sup _{\alpha} \inf _{\beta} \mathcal{L}_{\alpha \beta}^{t} u(x)$ can be considered. Characteristic properties of these operators can easily be derived as follows;

$$
\inf _{t \geq 1 / 2} \inf _{\alpha \beta} \mathcal{L}_{\alpha \beta}^{t} v(x) \leq \mathcal{I}^{ \pm}[u+v](x)-\mathcal{I}^{ \pm} u(x) \leq \sup _{t \geq 1 / 2} \sup _{\alpha \beta} \mathcal{L}_{\alpha \beta}^{t} v(x),
$$

where $\mathcal{I}^{ \pm} u$ are given by $\mathcal{I}^{+} u(x)=\sup _{t \geq 1 / 2} \mathcal{I}^{t} u(x)$ and $\mathcal{I}^{-} u(x)=\inf _{t \geq 1 / 2} \mathcal{I}^{t} u(x)$ (here we use $1 / 2$ on $t$ instead of 1 with certain technical reasons which can be found in the proof of 4.3.4 below).

A more general and better description of the fully nonlinear operators we want to deal with is the operator $\mathcal{J}$ for which (1.0.5) holds for some family of linear integro-differential operators $\mathcal{L}_{\alpha \beta}^{t}$. The idea is that the concept of ellipticity can be replaced by an estimate $\mathcal{M}_{\lambda, \Lambda}^{-} v(x) \leq \mathcal{J}[u+v](x)-\mathcal{J} u(x) \leq$ $\mathcal{M}_{\lambda, \Lambda}^{+} v(x)$, where $\mathcal{M}_{\lambda, \Lambda}^{-}$and $\mathcal{M}_{\lambda, \Lambda}^{+}$are the Pucci extremal operators [CC]. Then it is easy to see that $\mathcal{J}$ must be an elliptic second order differentiable 
operator. If instead we compare with suitable nonlocal extremal operators, we will have a concept of ellipticity for nonlocal operators $\mathcal{J}$. We shall give a precise definition in Section 2.2 (see Definition 2.2.1).

We now explain the natural Dirichlet problem for such nonlocal operators $\mathcal{I}^{ \pm}$. Let $\Omega$ be an open domain in $\mathbb{R}^{n}$. Given a function $g$ defined on $\mathbb{R}^{n} \backslash \Omega$, we want to find a function $u$ such that

$$
\begin{cases}\mathcal{I}^{ \pm} u(x)=0 & \text { for any } x \in \Omega, \\ u(x)=g(x) & \text { for } x \in \mathbb{R}^{n} \backslash \Omega .\end{cases}
$$

Note that the boundary condition is given not only on $\partial \Omega$ but also on the whole complement of $\Omega$. This is because of the nonlocal character of the operators $\mathcal{I}^{ \pm}$. From the stochastic point of view, it corresponds to the fact that a discontinuous Lèvy process can exit the domain $\Omega$ for the first time jumping to any point in $\mathbb{R}^{n} \backslash \Omega$.

In this paper, we shall concentrate mainly upon the regularity properties of viscosity solutions to an equation $\mathcal{I}^{ \pm} u(x)=0$. We shall briefly give a very general comparison principle from which existence of the solutions can be obtained in smooth domains. Since kernels of integro-differential operators are comparable to the kernel of the fractional Laplace operator $-(-\Delta)^{\sigma / 2}$, the theory we want to develop can be understood as a theory of viscosity solutions for fully nonlinear operators of fractional order.

1.1. The differences between local and nonlocal operators. Most of all, let us emphasize the main differences between local and nonlocal equations in terms of Harnack estimate and Hölder continuity.

- First, the nonnegativity of the solution of a local uniformly elliptic equation in a ball $B_{R}$ is enough to get a Harnack inequality in a smaller ball $B_{R / 2}$. For the nonlocal equation, there is a counterexample [BK1] that is nonnegative in $B_{R}$ but that has zero value in $B_{R / 2}$. It is due to the fact that there are influence for the values outside $B_{R}$, which should be controlled to have a Harnack inequality. So we impose that the solution is nonnegative in $\mathbb{R}^{n}$.

- Second, by applying Harnack inequalities on $\sup _{B_{R}} u-u(x)$ and $u(x)-$ $\inf _{B_{R}} u$, we are able to show the oscillation lemma and Hölder regularity of the solution of a local equation. However such method cannot be directly applicable due to the fact that we need nonnegativity of the solution in $\mathbb{R}^{n}$, not only $B_{R}$.

- The last interesting fact is that there is a discontinuous solution if the Kernel is allowed to oscillate between two different exponents $\sigma_{1}$ and $\sigma_{2}$, $[\mathrm{BBC} \mid$. The authors show that there is still a Harnack estimate if the radius of the ball has a positive lower bound, for example one.

1.2. History and New results. There are some known results about Harnack inequalities and Hölder estimates for integro-differential operators with positive symmetric kernels (see [J] for analytical proofs and [ $\overline{\mathrm{BBC}}]$, [BK1], [BK2],[BL], [KS], [SV] for probabilistic proofs). The estimates in all 
these previous results blow up as the index $\sigma$ of the operator approaches 2 . In this respect, they do not generalize to elliptic partial differential equations. However there is some known result on regularity results for fully nonlinear integro-differential equations associated with nonlinear integrodifferential operators with positive symmetric kernels which remain uniform as the index $\sigma$ of the operator approaches 2 (see [CS]). Therefore these results make the theory of integro-differential operators and elliptic differential operators become somewhat unified. There has been serious consideration on the concept of viscosity solution of fully nonlinear integrodifferential equations and their properties, [Ar, $\mathrm{AT}, \mathrm{BCI}$, BS, I, JK, $\mathrm{P}]$.

In this paper, we extend the important regularity results of Caffarelli and Silvestre [CS] on positive symmetric kernels with certain decay to those on certain positive (not necessarily symmetric) kernels including such symmetric kernels which remain uniform as the index $\sigma$ of the operator approaches 2 . In this occasion we can not expect any cancelation on the estimates contrary to the case of the symmetric kernel, which stirs up some difficulties in this problem.

Throughout this paper we would like to briefly present the necessary definitions and then prove some regularity estimates. Our results in this paper are the following.

- We introduce more general new class of operators to consider nonsymmetric case. It is invariant under translation and scaling, which are crucial properties used at [CS]. Still we are able to show standard porperties for viscosity solutions of the general nonlinear integro-differential equations.

- We show a new version of the nonlocal Alexandroff-Backelman-Pucci estimate for fully nonlinear integro-differential equations with possible nonsymmetric kernel. It contains the extra term caused by the nonsymmetry of kernel.

- We also give new proof for the construction of special functions to handle the nonsymmetry of the kernel. And then we show A Harnack inequality, Hölder regularity, and an interior $C^{1, \alpha}$-regularity result for certain fully nonlinear integro-differential equations.

1.3. Key Observations. Nonsymmetric case developes the following differences from the symmetric case and nontrivial difficulties. Key observations are the following:

- For the nonsymmetric case, $K(y)$ and $K(-y)$ can be chosen any of $\lambda /|y|^{n+\sigma}$ or $\Lambda /|y|^{n+\sigma}$. Therefore there could be an extra term $\int_{\mathbb{R}^{n}} \frac{\left|(\nabla u(x) \cdot y) \chi_{B_{t}}(y)\right|}{|y|^{n+\sigma}} d y$.

- The equation is not scaling invariant due to $\left|\chi_{B_{t}}(y)\right|$.

- Somehow the equation has a drift term, not only the diffusion term. The case $1<\sigma<2$ and the case $0<\sigma \leq 1$ require different technique due to the difference of the blow rate as $|y|$ approaches to zero and the decay rate as $|y|$ approaches to infinity. When $1<\sigma<2$, a controllable decay rate of kernel allows Hölder regularity in a larger class, which is invariant under 
an one-sided scaling i.e. if $u$ is a solution of the homogeneous equation, then so is $u_{\epsilon}(x)=\epsilon^{-\sigma} u(\epsilon x)$ for $0<\epsilon \leq 1$. Critical case $(\sigma=1)$ and supercritical case $(0<\sigma<1)$ have been studied in [KL] with different techniques due to the slow decay rate of the kernel as $|x| \rightarrow \infty$. It is noticeable that a gradient term has be considered as a lower order term $(1<\sigma<2)$, [I] , while our gradient effect comes from the diffusion term which is a main order term.

1.4. Outline of Paper. The outline of the paper is as follows. In Section 2.1. the appropriate definitions of subsolutions and supersolutions of fully nonlinear integro-differential equation in the viscosity sense shall be given. In the definitions, we shall allow some kind of discontinuities outside of the domain of the equation. In Section 2.2, we introduce more general concept of fully nonlinear integro-differential equation which is invariant under one-sided scaling . We define a nonlocal elliptic operator by comparing its increments with a suitable maximal operator. This definition is more general than (1.0.4). In Section 2.3, we study the stability properties of viscosity solutions given in the definition. A comparison principle is proven in Section 2.4 under very mild assumptions. We guess that one of the most nontrivial results in the paper is a nonlocal version of the Alexandroff-Backelman-Pucci estimate to be shown in Section 3. It has an extra term caused by the nonsymmetry of the kernel. It leads to regularity results for certain fully nonlinear integro-differential equations. In Section 4. we construct a special function, considering nonsymmetry of kernel, and obtain some pointwise estimates which shall be helpful in proving Hölder estimates in Section 5.2. In Section 5.1, we prove a Harnack inequality which plays an important role in analysis. We obtain the Hölder estimates in Section 5.2. Finally we show an interior $C^{1, \alpha}$-estimates in Section 5.3 .

\section{Viscosity Solutions}

2.1. Definitions. For our purpose, we shall restrict our attention to the operators $\mathcal{L}^{t}$ where the measure $m$ is given by a positive kernel $K$ which is not necessarily symmetric. That is to say, the operators $\mathcal{L}^{t}$ are given by

$$
\mathcal{L}^{t} u(x)=\text { p.v. } \int_{\mathbb{R}^{n}}\left[u(x+y)-u(x)-(\nabla u(x) \cdot y) \chi_{B_{t}}(y)\right] K(y) d y, t>0 .
$$

Then we see that $\mathcal{L}^{t} u(x)$ is well-defined provided that $u \in \mathrm{C}^{1,1}(x) \cap \mathrm{B}\left(\mathbb{R}^{n}\right)$ (refer to Definition 2.1.3 for the definition of $C^{1,1}(x)$ ) where $B\left(\mathbb{R}^{n}\right)$ denotes the family of all real-valued bounded functions defined on $\mathbb{R}^{n}$. To simplify the notation, we write $\mu_{t}(u, x, y)=u(x+y)-u(x)-(\nabla u(x) \cdot y) \chi_{B_{t}}(y), t>0$. Then the expression for $\mathcal{L}^{t}$ may shortly be written as

$$
\mathcal{L}^{t} u(x)=\int_{\mathbb{R}^{n}} \mu_{t}(u, x, y) K(y) d y
$$


In particular, for $t>0$, we consider the class $\mathfrak{L}_{t}$ of the operators $\mathcal{L}^{t}$ associated with the measures $m$ given by positive kernels $K \in \mathcal{K}_{0}$ satisfying that

$$
(2-\sigma) \frac{\lambda}{|y|^{n+\sigma}} \leq K(y) \leq(2-\sigma) \frac{\Lambda}{|y|^{n+\sigma}}, 1<\sigma<2 .
$$

In what follows, our main concern shall be on the nonlinear integrodifferential operators which have the form like (1.0.5) where we think that each $\mathcal{L}_{\alpha \beta}^{t} \in \mathfrak{I}_{t}$ has a kernel $K_{\alpha \beta} \in \mathcal{K}_{0}$ satisfying (2.1.3). The minimum assumption so that $\mathcal{I}^{ \pm} u$ are well-defined is that every kernel $K_{\alpha \beta}$ must satisfy the following integrability condition in a uniform way; more precisely, if we set $K(y)=\sup _{\alpha \beta} K_{\alpha \beta}(y)$, then

$$
\int_{\mathbb{R}^{n}}\left(|y|^{2} \wedge 1\right) K(y) d y<\infty .
$$

We say that $P$ is a paraboloid of opening $M$ if

$$
P(x)=\ell_{0}+\ell(x) \pm \frac{M}{2}|x|^{2}
$$

where $M$ is a positive constant, $\ell_{0}$ is real constant and $\ell$ is a linear function. Then $P$ is called convex when we have + in (2.1.5) and concave when we have - in (2.1.5). Let $\Omega \subset \mathbb{R}^{n}$ be a bounded domain. Given two semicontinuous functions $u, v$ defined on an open subset $U \subset \Omega$ and a point $x_{0} \in U$, we say that $v$ touches $u$ by above at $x_{0} \in U$ if $u\left(x_{0}\right)=v\left(x_{0}\right)$ and $u(x) \leq v(x)$ for any $x \in U$. Similarly, we say that $v$ touches $u$ by below at $x_{0} \in U$ if $u\left(x_{0}\right)=v\left(x_{0}\right)$ and $u(x) \geq$ $v(x)$ for any $x \in U$. For a semicontinuous function $u$ on $\Omega$ and an open subset $U$ of $\Omega$, we define $\Theta^{+}(u, U)\left(x_{0}\right)$ to be the infimum of all positive constants $M$ for which there is a convex paraboloid of opening $M$ that touches $u$ by above at $x_{0} \in U$. Also we define $\Theta^{+}(u, U)\left(x_{0}\right)=\infty$ if no such constant $M$ exists. Similarly, we define $\Theta^{-}(u, U)\left(x_{0}\right)$ to be the infimum of all positive constants $M$ for which there is a concave paraboloid of opening $M$ that touches $u$ by below at $x_{0} \in U$, and also we define $\Theta^{-}(u, U)\left(x_{0}\right)=\infty$ if no such constant $M$ exists. Finally we set $\Theta(u, U)\left(x_{0}\right)=\max \left\{\Theta^{+}(u, U)\left(x_{0}\right), \Theta^{-}(u, U)\left(x_{0}\right)\right\} \leq \infty$. For these definitions, the readers can refer to [CC].

Definition 2.1.1. Let $\Omega \subset \mathbb{R}^{n}$ be a bounded domain and let $u: \Omega \rightarrow \mathbb{R}$ be a semicontinuous function. Given $x_{0} \in \Omega$, we say that $u$ is $C_{ \pm}^{1,1}$ at $x_{0}$ (resp. $C^{1,1}$ at $\left.x_{0}\right)$ if $\Theta^{ \pm}(u, U)\left(x_{0}\right)<\infty$ ( resp. $\left.\Theta(u, U)\left(x_{0}\right)<\infty\right)$ for some open neighborhood $U$ of $x_{0}$ and we write $u \in C^{1,1}\left[x_{0}\right]$ if $\Theta(u, U)\left(x_{0}\right)<\infty$. Given a fixed $\epsilon \in(0,1)$, we set $\Theta(u, \epsilon)(x)=\Theta\left(u, \Omega \cap B_{\epsilon}(x)\right)$ for $x \in \Omega$ and we write $u \in C^{1,1}[\Omega]$ if $\sup _{x \in \Omega} \Theta(u, \epsilon)(x) \fallingdotseq \Theta_{\Omega}[u]<\infty$.

Remark 2.1.2. (a) We note that if $u \in C^{1,1}\left[x_{0}\right]$ then $u$ is differentiable at $x_{0}$ because $u$ lies between two tangent paraboloids in an open neighborhood of $x_{0}$.

(b) In fact, the number $\Theta_{\Omega}[u]$ in Definition 2.1.1 depends upon $\epsilon$. But existence of $\epsilon$ so that $\Theta_{\Omega}[u]<\infty$ is enough, because $\Theta_{\Omega}[u]$ decreases as $\epsilon$ approaches 0 . 
Definition 2.1.3. A function $u: \mathbb{R}^{n} \rightarrow \mathbb{R}$ is said to be $C^{1,1}$ at a point $x \in \mathbb{R}^{n}$ (we write $u \in C^{1,1}(x)$ ), if there exist some vector $v \in \mathbb{R}^{n}, r_{0}>0$ and $M>0$ such that

$$
|u(x+y)-u(x)-v \cdot y| \leq M|y|^{2} \text { for any } y \in B_{r_{0}} .
$$

We write $u \in C^{1,1}(U)$ if $u \in C^{1,1}(x)$ for any $x \in U$ and the constant $M$ in (2.1.6) is independent of $x$, where $U$ is an open subset of $\mathbb{R}^{n}$.

Remark 2.1.4. (a) Such vector $v$ exists uniquely and moreover $v=\nabla u(x)$.

(b) If $u \in C^{1,1}[x]$ for $x \in \Omega$, then we easily see that $u \in C^{1,1}(x)$. Moreover, it is easy to show that the converse holds. Thus we conclude that $C^{1,1}[\Omega]=C^{1,1}(\Omega)$.

For $x \in \Omega$ and a function $u: \mathbb{R}^{n} \rightarrow \mathbb{R}$ which is semicontinuous on $\bar{\Omega}$, we say that $\varphi$ belongs to the function class $\mathrm{C}_{\Omega}^{2}(u ; x)^{+}\left(\right.$resp. $\left.\mathrm{C}_{\Omega}^{2}(u ; x)^{-}\right)$and we write $\varphi \in \mathrm{C}_{\Omega}^{2}(u ; x)^{+}$(resp. $\left.\varphi \in \mathrm{C}_{\Omega}^{2}(u ; x)^{-}\right)$if there are an open neighborhood $U \subset \Omega$ of $x$ and $\varphi \in C^{2}(U)$ such that $\varphi(x)=u(x)$ and $\varphi>u$ (resp. $\varphi<u$ ) on $U \backslash\{x\}$. We note that geometrically $u-\varphi$ having a local maximum at $x$ in $\Omega$ is equivalent to $\varphi \in \mathrm{C}_{\Omega}^{2}(u ; x)^{+}$and $u-\varphi$ having a local minimum at $x$ in $\Omega$ is equivalent to $\varphi \in \mathrm{C}_{\Omega}^{2}(u ; x)^{-}$. For $x \in \Omega$ and $\varphi \in \mathrm{C}_{\Omega}^{2}(u ; x)^{ \pm}$, we write

$$
\mu_{t}(u, x, y ; \nabla \varphi)=u(x+y)-u(x)-(\nabla \varphi(x) \cdot y) \chi_{B_{t}}(y), t>0,
$$

and the expression for $\mathcal{L}_{\alpha \beta}^{t} u(x ; \nabla \varphi)$ and $\mathcal{I}^{t} u(x ; \nabla \varphi)$ may be written as

$$
\begin{aligned}
\mathcal{L}_{\alpha \beta}^{t} u(x ; \nabla \varphi) & =\int_{\mathbb{R}^{n}} \mu_{t}(u, x, y ; \nabla \varphi) K_{\alpha \beta}(y) d y, \\
\mathcal{I}^{t} u(x ; \nabla \varphi) & =\inf _{\beta} \sup _{\alpha} \mathcal{L}_{\alpha \beta}^{t} u(x ; \nabla \varphi),
\end{aligned}
$$

where $K_{\alpha \beta} \in \mathcal{K}_{0}$. We set $\mathcal{I}^{+} u(x ; \nabla \varphi)=\sup _{t \geq 1 / 2} \mathcal{I}^{t} u(x ; \nabla \varphi)$ and $\mathcal{I}^{-} u(x ; \nabla \varphi)=$ $\inf _{t \geq 1 / 2} \mathcal{I}^{t} u(x ; \nabla \varphi)$. We note that if $u \in C^{1,1}(x)$, then $\mu_{t}(u, x, y ; \nabla \varphi)=\mu_{t}(u, x, y)$, $\mathcal{L}_{\alpha \beta}^{t} u(x ; \nabla \varphi)=\mathcal{L}_{\alpha \beta}^{t} u(x)$ and $\mathcal{I}^{t} u(x ; \nabla \varphi)=\mathcal{I}^{t} u(x)$ for $x \in \Omega$ and $\varphi \in C_{\Omega}^{2}(u ; x)^{ \pm}$.

Definition 2.1.5. Let $f: \mathbb{R}^{n} \rightarrow \mathbb{R}$ be a function. Then a function $u: \mathbb{R}^{n} \rightarrow \mathbb{R}$ which is upper (lower) semicontinuous on $\bar{\Omega}$ is said to be a viscosity subsolution (viscosity supersolution) of an equation $\mathcal{J} u=f$ on $\Omega$ and we write $\mathcal{J} u \geq f$ $(\mathcal{J} u \leq f)$ on $\Omega$ in the viscosity sense, if for any $x \in \Omega$ there is some open neighborhood $U$ of $x$ with $U \subset \Omega$ such that $\mathcal{J} v(x)$ is well-defined and $\mathcal{J} v(x) \geq f(x)$ $(\mathcal{J} v(x) \leq f(x))$ for $v=\varphi \chi_{U}+u \chi_{\mathbb{R}^{n} \backslash U}$ whenever $\varphi \in C^{2}(U)$ satisfying $\varphi(x)=u(x)$ and $\varphi>u(\varphi<u)$ on $U \backslash\{x\}$ exists. Also a function $u$ which is both a viscosity subsolution and a viscosity supersolution of the equation $\mathcal{J} u=f$ on $\Omega$ is said to be a viscosity solution to $\mathcal{J} u=f$ on $\Omega$.

Remark 2.1.6. Definition 2.1.5 is essentially the same as Definition 2 in [BI].

Instead of test functions $\varphi \in \mathrm{C}^{2}$ given in the above, functions $\varphi$ which are $C^{1,1}$ only at the contact point $x$ could be used. This is a larger set of test functions, so that a priori it may provide a stronger concept of solution. In Section 2.3. we shall show that the two approaches are actually equivalent. 
Theorem 2.1.7. Let $f: \mathbb{R}^{n} \rightarrow \mathbb{R}$ be a function. Then we have the followings:

(a) If $u: \mathbb{R}^{n} \rightarrow \mathbb{R}$ is a function which is upper semicontinuous on $\bar{\Omega}$, then $\mathcal{I}^{ \pm} u \geq f$ on $\Omega$ in the viscosity sense if and only if $\mathcal{I}^{ \pm} u(x ; \nabla \varphi)$ is well-defined and

$$
\mathcal{I}^{ \pm} u(x ; \nabla \varphi) \geq f(x) \text { for any } x \in \Omega \text { and } \varphi \in \mathrm{C}_{\Omega}^{2}(u ; x)^{+} .
$$

(b) If $u: \mathbb{R}^{n} \rightarrow \mathbb{R}$ is a function which is lower semicontinuous on $\bar{\Omega}$, then $\mathcal{I}^{ \pm} u \leq f$ on $\Omega$ in the viscosity sense if and only if $\mathcal{I}^{ \pm} u(x ; \nabla \varphi)$ is well-defined and

$$
\mathcal{I}^{ \pm} u(x ; \nabla \varphi) \leq f(x) \text { for any } x \in \Omega \text { and } \varphi \in \mathrm{C}_{\Omega}^{2}(u ; x)^{-} .
$$

(c) If $u: \mathbb{R}^{n} \rightarrow \mathbb{R}$ is a function which is continuous on $\bar{\Omega}$, then $u$ is a viscosity solution to $\mathcal{I}^{ \pm} u=f$ on $\Omega$ if and only if it satisfies both (2.1.7) and (2.1.8).

Proof. Since (b) and (c) can be obtained by the similar way to the proof of (a), we have only to prove the equivalence (a) for $\mathcal{I}^{-}$; similarly for $\mathcal{I}^{+}$.

Assume that $\mathcal{I}^{-} u \geq f$ on $\Omega$ in the viscosity sense. Fix any $x \in \Omega$ and take any $\varphi \in \mathrm{C}_{\Omega}^{2}(u ; x)^{+}$. Then there is an open neighborhood $U \subset \Omega$ of $x$ such that $u(x)=\varphi(x)$ and $\varphi>u$ on $U \backslash\{x\}$. For $0<s<\mathrm{d}(x, \partial U)$, we set $u_{s}=\varphi \chi_{B_{s}(x)}+u \chi_{\mathbb{R}^{n} \backslash B_{s}(x)}$ and $v=\varphi \chi_{U}+u \chi_{\mathbb{R}^{n} \backslash U}$. Then, for any $t \geq 1 / 2$ and any $\beta$, there is some $\alpha$ such that $\mathcal{L}_{\alpha \beta}^{t} v(x) \geq \mathcal{L}_{\alpha \beta}^{t} u_{s}(x) \geq f(x)$. By the Lebesgue's dominated convergence theorem, taking $s \downarrow 0$ we conclude that for any $t \geq 1 / 2$ and any $\beta$ there is some $\alpha$ such that $\mathcal{L}_{\alpha \beta}^{t} v(x) \geq \mathcal{L}_{\alpha \beta}^{t} u(x ; \nabla \varphi) \geq f(x)$. Therefore $\mathcal{I}^{-} u(x ; \nabla \varphi)$ is well-defined and $\mathcal{I}^{-} u(x ; \nabla \varphi) \geq f(x)$.

Conversely, suppose that (2.1.7) holds. Let $u: \mathbb{R}^{n} \rightarrow \mathbb{R}$ be a function which is upper semicontinuous on $\bar{\Omega}$. Fix any $x \in \Omega$ and take a function $\varphi \in \mathrm{C}^{2}(U)$ satisfying that $\varphi(x)=u(x)$ and $\varphi>u$ on $U \backslash\{x\}$ where $U$ is an open neighborhood $U \subset \Omega$ of $x$. Then $\varphi \in C_{\Omega}^{2}(u ; x)^{+}$. If we set $v=$ $\varphi \chi_{U}+u \chi_{\mathbb{R}^{n}} \backslash U$, then for any $t \geq 1 / 2$ and any $\beta$ there is some $\alpha$ such that $\mathcal{L}_{\alpha \beta}^{t} v(x) \geq \mathcal{L}_{\alpha \beta}^{t} u(x ; \nabla \varphi) \geq f(x)$, and thus $\mathcal{I}^{-} v(x) \geq f(x)$. Hence we conclude that $\mathcal{I}^{-} u \geq f$ on $\Omega$ in the viscosity sense.

2.2. Maximal operators. In (1.0.3) and (1.0.4), we considered the supremum or an inf-sup of a collection of linear integro-differential operators. Let us consider a class $\mathfrak{L}$ of linear operators which includes the class $\mathfrak{L}_{0}=\bigcup_{t \geq 1 / 2} \mathfrak{L}_{t}$ given in Section 2.1. The maximal operator and the minimal operator with respect to $\mathfrak{L}$ are defined by

$$
\mathcal{M}_{\mathfrak{L}}^{+} u(x)=\sup _{\mathcal{L} \in \mathfrak{L}} \mathcal{L} u(x) \text { and } \mathcal{M}_{\mathfrak{Q}}^{-} u(x)=\inf _{\mathcal{L} \in \mathfrak{L}} \mathcal{L} u(x) .
$$

For a function $u: \mathbb{R}^{n} \rightarrow \mathbb{R}$ semicontinuous on $\bar{\Omega}$ and $\varphi \in C_{\Omega}^{2}(u ; x)^{ \pm}$, we set

$$
\mathcal{M}_{\mathfrak{I}_{0}}^{+} u(x ; \nabla \varphi)=\sup _{t \geq 1 / 2} \mathcal{M}_{\mathfrak{S}_{t}}^{+} u(x ; \nabla \varphi) \text { and } \mathcal{M}_{\mathfrak{I}_{0}}^{-} u(x ; \nabla \varphi)=\inf _{t \geq 1 / 2} \mathcal{M}_{\mathfrak{I}_{t}}^{-} u(x ; \nabla \varphi),
$$

where $\mathcal{M}_{\mathfrak{Q}_{t}}^{+} u(x)=\sup _{\mathcal{L}_{\mathcal{E}} \mathfrak{Q}_{t}} \mathcal{L} u(x)$ and $\mathcal{M}_{\mathfrak{L}_{t}}^{-} u(x)=\inf _{\mathcal{L} \in \mathfrak{I}_{t}} \mathcal{L} u(x)$. Then we see that $\mathcal{M}_{\mathfrak{I}_{0}}^{-} u(x ; \nabla \varphi) \leq \mathcal{I}^{ \pm} u(x ; \nabla \varphi) \leq \mathcal{M}_{\mathfrak{I}_{0}}^{+} u(x ; \nabla \varphi)$, and $\mathcal{M}_{\mathfrak{I}_{0}}^{ \pm} u(x ; \nabla \varphi)$ have the 
following simple forms;

$$
\begin{aligned}
& \mathcal{M}_{\mathfrak{L}_{0}}^{+} u(x ; \nabla \varphi)=(2-\sigma) \int_{\mathbb{R}^{n}} \frac{\Lambda \bar{\mu}^{+}(u, x, y ; \nabla \varphi)-\lambda \underline{\mu}^{-}(u, x, y ; \nabla \varphi)}{|y|^{n+\sigma}} d y, \\
& \mathcal{M}_{\mathfrak{Q}_{0}}^{-} u(x ; \nabla \varphi)=(2-\sigma) \int_{\mathbb{R}^{n}} \frac{\lambda \underline{\mu}^{+}(u, x, y ; \nabla \varphi)-\Lambda \bar{\mu}^{-}(u, x, y ; \nabla \varphi)}{|y|^{n+\sigma}} d y,
\end{aligned}
$$

where $\bar{\mu}^{+}, \underline{\mu}^{+}, \bar{\mu}^{-}$and $\underline{\mu}^{-}$are given by

$\bar{\mu}^{ \pm}(u, x, y ; \nabla \varphi)=\sup _{t \geq 1 / 2} \mu_{t}^{ \pm}(u, x, y ; \nabla \varphi)$ and $\underline{\mu}^{ \pm}(u, x, y ; \nabla \varphi)=\inf _{t \geq 1 / 2} \mu_{t}^{ \pm}(u, x, y ; \nabla \varphi)$.

We note that if $u \in C^{1,1}(x)$, then $\mathcal{M}_{\mathfrak{I}_{0}}^{ \pm} u(x ; \nabla \varphi)=\mathcal{M}_{\mathfrak{I}_{0}}^{ \pm} u(x)$. We shall use these maximal and minimal operators to obtain regularity estimates. The factor $(2-\sigma)$ is important when $\sigma \rightarrow 2$, because we need such factor if we want to obtain second order partial differential equations as limits of linear integro-differential equations. In terms of the regularity, we need the factor $(2-\sigma)$ for the estimates not to blow up as $\sigma \rightarrow 2$.

Let $K(x)=\sup _{\alpha} K_{\alpha}(x)$ where $K_{\alpha}$ 's are all the kernels of all operators in $\mathfrak{L}$. Instead of (2.1.4), for any class $\mathfrak{L}$ we shall assume that

$$
\int_{\mathbb{R}^{n}}\left(|y|^{2} \wedge 1\right) K(y) d y<\infty
$$

Using the extremal operators, we provide a general definition of ellipticity for nonlocal equations. The following is a kind of operators of which the regularity result shall be obtained in this paper.

Definition 2.2.1. Let $\mathfrak{Q}$ be a class of linear integro-differential operators. Assume that (2.2.3) holds for $\mathfrak{R}$. Then we say that an operator $\mathcal{J}$ is elliptic with respect to $\mathfrak{L}$, if it satisfies the following properties:

(a) $\mathcal{J} u(x)$ is well-defined for any $u \in \mathrm{C}^{1,1}[x] \cap \mathrm{B}\left(\mathbb{R}^{n}\right)$.

(b) $\mathcal{J} u$ is continuous on an open set $\Omega \subset \mathbb{R}^{n}$, whenever $u \in C^{1,1}[\Omega] \cap \mathrm{B}\left(\mathbb{R}^{n}\right)$.

(c) If $u, v \in \mathrm{C}^{1,1}[x] \cap \mathrm{B}\left(\mathbb{R}^{n}\right)$, then we have that

$$
\mathcal{M}_{\mathfrak{I}}^{-}[u-v](x) \leq \mathcal{J} u(x)-\mathcal{J} v(x) \leq \mathcal{M}_{\mathfrak{Q}}^{+}[u-v](x) .
$$

We shall show that any operator as in (1.0.5) is elliptic with respect to any class containing all the operators $\mathcal{L}_{\alpha \beta}^{t}$ as long as the condition (2.1.4) is satisfied (see Lemma 2.2.2 and Lemma 2.3.2).

Lemma 2.2.2. Let $\mathcal{I}^{ \pm}$be the operators as in (1.0.5) so that (2.1.4) holds for every $K_{\alpha \beta}$ and let $\mathfrak{L}$ be any collection of linear integro-differential operators. If $\mathfrak{L}_{0} \subset \mathfrak{L}$ where $\mathfrak{L}_{0}=\bigcup_{t \geq 1 / 2} \mathfrak{L}_{t}$, then we have that

$$
\mathcal{M}_{\mathfrak{L}}^{-}[u-v](x) \leq \mathcal{I}^{ \pm} u(x)-\mathcal{I}^{ \pm} v(x) \leq \mathcal{M}_{\mathfrak{L}}^{+}[u-v](x)
$$

for any $u, v \in \mathrm{C}^{1,1}[x] \cap \mathrm{B}\left(\mathbb{R}^{n}\right)$. 
Proof. It can be shown in a similar way as in [CS].

Definition 2.1.3 is not set up to evaluate the operator $\mathcal{J}$ in the original function $u$. Whenever a smooth function $\varphi$ touches $u$ from above, we can always construct a test function $v \in C^{1,1}[x]$ to evaluate $\mathcal{J}$. It is very interesting that if $\mathcal{J}$ is any nonlinear operator which is formulated by an inf-sup (or a sup-inf) of linear operators satisfying (2.1.3) and (2.1.4), then without constructing such a test function $\mathcal{J}$ can be evaluated classically in $u$ at those points $x$ where $u$ can be touched by above with a $C^{2}$ function. This interesting fact is proved in the next lemma.

Lemma 2.2.3. Let $\mathcal{I}^{ \pm}$be the operator as in (1.0.5) so that (2.1.4) holds for every $K_{\alpha \beta}$ and let $u \in \mathrm{B}\left(\mathbb{R}^{n}\right)$ be a viscosity subsolution to $\mathcal{I}^{ \pm} u=$ f on $\Omega$. If $u \in C^{1,1}[x]$ for a point $x \in \Omega$, then $\mathcal{I}^{ \pm} u(x)$ is defined in the classical sense and $\mathcal{I}^{ \pm} u(x) \geq f(x)$.

Proof. It can be obtained in a similar way as in [CS].

In the next theorem, we shall obtain a result on the operators $\mathcal{M}_{\mathfrak{Q}_{0}}^{ \pm}$which is similar to Theorem 2.1.7. The proof is almost the same as that of Theorem 2.1.7 and so we will just write out the statement without detailed proof.

Theorem 2.2.4. Let $f: \mathbb{R}^{n} \rightarrow \mathbb{R}$ be a function. Then we have the followings:

(a) If $u: \mathbb{R}^{n} \rightarrow \mathbb{R}$ is a function which is upper semicontinuous on $\bar{\Omega}$, then $u$ is a viscosity subsolution to $\mathcal{M}_{\mathfrak{L}_{0}}^{+} u=f$ on $\Omega$ if and only if $\mathcal{M}_{\mathbb{\Omega}_{0}}^{+} u(x ; \nabla \varphi)$ is well-defined and $\mathcal{M}_{\mathfrak{\Omega}_{0}}^{+} u(x ; \nabla \varphi) \geq f(x)$ for any $x \in \Omega$ and any $\varphi \in \mathrm{C}_{\Omega}^{2}(u ; x)^{+}$.

(b) If $u: \mathbb{R}^{n} \rightarrow \mathbb{R}$ is a function which is lower semicontinuous on $\bar{\Omega}$, then $u$ is a viscosity supersolution to $\mathcal{M}_{\mathfrak{\Omega}_{0}}^{-} u=f$ on $\Omega$ if and only if $\mathcal{M}_{\mathbb{\Omega}_{0}}^{-} u(x ; \nabla \varphi)$ is well-defined and $\mathcal{M}_{\mathfrak{\Omega}_{0}}^{-} u(x ; \nabla \varphi) \leq f(x)$ for any $x \in \Omega$ and any $\varphi \in \mathrm{C}_{\Omega}^{2}(u ; x)^{-}$.

2.3. Stability properties. In this section, we obtain a few technical properties of the operators $\mathcal{I}^{ \pm}$as in (1.0.5). First we shall show that if $u \in$ $\mathrm{C}^{1,1}[\Omega] \cap \mathrm{B}\left(\mathbb{R}^{n}\right)$, then $\mathcal{I}^{ \pm} u$ are continuous on $\Omega$. As mentioned in the previous sections, it is necessary to justify that the operators of the form (1.0.3) and (1.0.4) satisfy the conditions of Definition 2.2.1. Next we shall show that our notion of viscosity solutions allows to touch with solutions which are only punctually $C^{1,1}$ instead of $C^{2}$ in a neighborhood of the point. Then we shall show the important stability property of viscosity solutions given in Definition 2.1.3. We now start with several technical lemmas.

Lemma 2.3.1. [CS] Let $\left\{h_{\alpha}\right\}$ be a family of functions such that $\left|h_{\alpha}(x)\right| \leq h(x)$ for $h \in L^{1}\left(\mathbb{R}^{n}\right)$. If $f \in L^{\infty}\left(\mathbb{R}^{n}\right)$, then the family $\left\{f * h_{\alpha}\right\}$ is uniformly equicontinuous on every compact subsets of $\mathbb{R}^{n}$.

When we gave the definition of viscosity solutions in Section 2.1, we used $\mathrm{C}^{2}$ test functions. Now we show that it is equivalent to use punctually $\mathrm{C}^{1,1}$ functions. 
Lemma 2.3.2. Let $\left\{\mathcal{I}^{t}\right\}_{t \geq 1 / 2}$ be the family of operators as in (1.0.3) and (1.0.4) satisfying (2.1.4) and let $\Omega \subset \mathbb{R}^{n}$ be a bounded domain. If $u \in C^{1,1}[\Omega] \cap \mathrm{B}\left(\mathbb{R}^{n}\right)$, then $\mathcal{I}^{ \pm} u$ are continuous on $\Omega$.

Proof. Fix any $t>0$. Then we have only to prove that the family $\left\{\mathcal{L}_{\alpha \beta}^{t} u\right\}$ is equicontinuous on $\Omega$. We set $K(x)=\sup _{\alpha \beta} K_{\alpha \beta}(x)$ as in (2.1.4). Take any $\epsilon>0$ and $x_{0} \in \Omega$. Since $\Theta_{\Omega}[u]<\infty$, we have that $\left|\mu_{t}(u, x, y)\right|<\Theta_{\Omega}[u]|y|^{2}$, whenever $x \in \Omega$ and $|y|<t_{1} \fallingdotseq \operatorname{dist}(x, \partial \Omega) \wedge t$. Then choose some sufficiently small $t_{0} \in\left(0, t_{1}\right)$ so that

$$
\int_{B_{t_{0}}} \Theta_{\Omega}[u]|y|^{2} K(y) d y<\epsilon / 3 .
$$

Now we have that

$$
\begin{aligned}
\mathcal{L}_{\alpha \beta}^{t} u(x) & =\int_{B_{t_{0}}} \mu_{t}(u, x, y) K_{\alpha \beta}(y) d y+\int_{\mathbb{R}^{n} \backslash B_{t_{0}}} \mu_{t}(u, x, y) K_{\alpha \beta}(y) d y \\
& \fallingdotseq \mathcal{L}_{\alpha \beta}^{t, 0} u(x)+\mathcal{L}_{\alpha \beta}^{t, 1} u(x) .
\end{aligned}
$$

From (2.3.1), we easily obtain that $\left|\mathcal{L}_{\alpha \beta}^{t, 0} u(x)\right| \leq \int_{B_{t_{0}}} \Theta_{\Omega}[u]|y|^{2} K(y) d y<\epsilon / 3$ for any $\alpha, \beta$, whenever $x \in \Omega$. We also write

$$
\begin{aligned}
\mathcal{L}_{\alpha \beta}^{t, 1} u(x) & =u * h_{\alpha \beta}(x)-\left(\int_{\mathbb{R}^{n}} h_{\alpha \beta}(y) d y\right) u(x)-\int_{B_{t}}[\nabla u(x) \cdot y] h_{\alpha \beta}(y) d y \\
& \fallingdotseq \mathcal{L}_{\alpha \beta}^{t, 2} u(x)+\mathcal{L}_{\alpha \beta}^{t, 3} u(x)+\mathcal{L}_{\alpha \beta}^{t, 4} u(x)
\end{aligned}
$$

where $h_{\alpha \beta}(y)=K_{\alpha \beta}(y) \chi_{\mathbb{R}^{n} \backslash B_{t_{0}}}(y)$. Since $\Theta_{\Omega}[u]<\infty$ and $\nabla u$ is Lipschitz continuous on $\Omega$ by standard analysis, we have that

$$
\left|\mathcal{L}_{\alpha \beta}^{t, 4} u(x)-\mathcal{L}_{\alpha \beta}^{t, 4} u\left(x_{0}\right)\right| \leq\left(\int_{\mathbb{R}^{n}} h_{\alpha \beta}(y)|y| d y\right) \Theta_{\Omega}[u]\left|x-x_{0}\right|
$$

whenever $x \in B_{s}\left(x_{0}\right)$ and $B_{s}\left(x_{0}\right) \subset \Omega$. Since $\sup _{\alpha, \beta} \int_{\mathbb{R}^{n}} h_{\alpha \beta}(y)|y| d y<\infty$ (by (2.1.4) ) and $u \in C^{1,1}[\Omega]$, by Lemma 2.3.1, (2.3.3) and (2.3.4) there exists some sufficiently small $\delta=\delta(\varepsilon)>0$ such that

$$
\left|\mathcal{L}_{\alpha \beta}^{t, 1} u(x)-\mathcal{L}_{\alpha \beta}^{t, 1} u\left(x_{0}\right)\right|<\epsilon / 3
$$

for any $\alpha, \beta$, and $t \geq 1 / 2$, whenever $x \in \Omega$ and $\left|x-x_{0}\right|<\delta$. Thus it follows from (2.3.2) and (2.3.5) that

$$
\left|\mathcal{L}_{\alpha \beta}^{t} u(x)-\mathcal{L}_{\alpha \beta}^{t} u\left(x_{0}\right)\right| \leq\left|\mathcal{L}_{\alpha \beta}^{t, 0} u(x)\right|+\left|\mathcal{L}_{\alpha \beta}^{t, 0} u\left(x_{0}\right)\right|+\left|\mathcal{L}_{\alpha \beta}^{t, 1} u(x)-\mathcal{L}_{\alpha \beta}^{t, 1} u\left(x_{0}\right)\right|<\epsilon
$$

for any $\alpha, \beta$, and $t \geq 1 / 2$, whenever $x \in \Omega$ and $\left|x-x_{0}\right|<\delta$. Hence this implies that $\left|\mathcal{I}^{ \pm} u(x)-\mathcal{I}^{ \pm} u\left(x_{0}\right)\right|<\epsilon$, whenever $x \in \Omega$ and $\left|x-x_{0}\right|<\delta$. Therefore we complete the proof.

When we gave the definition of viscosity solutions in Section 2.1, we used $C^{2}$ test functions. We show it is equivalent to use punctually $C^{1,1}$ functions. 
Lemma 2.3.3. Let $\mathcal{J}$ be elliptic with respect to a class $\mathfrak{Q}$ in the sense of Definition 2.2.1 Assume that $u: \mathbb{R}^{n} \rightarrow \mathbb{R}$ is a viscosity subsolution to $\mathcal{J} u=f$ on $\Omega$ and $\varphi \in C^{1,1}[x] \cap \mathrm{B}\left(\mathbb{R}^{n}\right)$ for $x \in \Omega$. If $\varphi$ touches globally $u$ from above at $x$, then $\mathcal{J} \varphi(x)$ is defined in the classical sense and $\mathcal{J} \varphi(x) \geq f(x)$.

Proof. It can be shown in a similar way as in [CS].

One of the most useful properties of viscosity solutions is their stability property under uniform limits on compact sets. We shall prove a slightly stronger result that the notion of viscosity subsolution (supersolution) is stable with respect to the natural limits for upper (lower) semicontinuous functions. This type of limit is well-known and usually called $\Gamma$-limit. It was originally called as the celebrated half relaxed limit techniques by Barles and Perthame, but we are going to follow the similar definitions at [CS].

Definition 2.3.4. A sequence $\left\{u_{k}\right\}$ of lower semicontinuous functions is said to $\Gamma$-converges to $u$ on a set $\Omega \subset \mathbb{R}^{n}$ if $(a)$ for any sequence $\left\{x_{k}\right\} \subset \Omega$ with $\lim _{k \rightarrow \infty} x_{k}=x, \liminf _{k \rightarrow \infty} u_{k}\left(x_{k}\right) \geq u(x)$ and $(b)$ for any $x \in \Omega$, there is a sequence $\left\{x_{k}\right\} \subset \Omega$ with $\lim _{k \rightarrow \infty} x_{k}=x$ such that $\lim _{k \rightarrow \infty} u_{k}\left(x_{k}\right)=u(x)$.

Remark. (a) A uniformly convergent sequence $\left\{u_{k}\right\}$ converges in the $\Gamma$ sense.

(b) If $\left\{u_{k}\right\} \Gamma$-converges to $u$ on $\Omega$ and $u$ has a strict local minimum at $x$ then there is a sequence $\left\{x_{k}\right\}$ with $\lim _{k \rightarrow \infty} x_{k}=x$ such that each $u_{k}$ has a local minimum at $x_{k}$ (see [GD]).

(c) If $\left\{u_{k}\right\} \Gamma$-converges to $u$ on $\Omega$, then $\left\{u_{k}-\varphi\right\} \Gamma$-converges to $u-\varphi$ on $\Omega$ where $\varphi \in C(\Omega)$.

(d) From (b) and (c), we can get that if $\left\{u_{k}\right\} \Gamma$-converges to $u$ on $\Omega$ and $u-\varphi$ has a strict local minimum at $x$ where $\varphi \in \mathrm{C}(\Omega)$, then there is a sequence $\left\{x_{k}\right\}$ with $\lim _{k \rightarrow \infty} x_{k}=x$ such that each $u_{k}-\varphi$ has a local minimum at $x_{k}$.

Lemma 2.3.5. Let $\mathcal{J}$ be elliptic in the sense of Definition 2.2.1 If $\left\{u_{k}\right\} \subset \mathrm{B}\left(\mathbb{R}^{n}\right)$ is a sequence of viscosity supersolutions $u_{k}$ to $\mathcal{J} u_{k}=f_{k}$ on $\Omega$ such that

(a) $\left\{u_{k}\right\} \Gamma$-converges to $u$ in $\Omega,(b)\left\{u_{k}\right\}$ converges to $u$ a.e. on $\mathbb{R}^{n}$ and

(c) $\left\{f_{k}\right\}$ converges to $f$ locally uniformly on $\Omega$, then $u$ is a viscosity supersolution to $\mathcal{J} u=f$ on $\Omega$.

Proof. It can be done with minor changes in a similar way as in [CS].

We just obtained the stability property of supersolutions under $\Gamma$-limits. For the corresponding result for subsolutions as in the following lemma, we would also consider the natural limit in the space of upper semicontinuous functions which is the same as the $\Gamma$-convergence of $-u_{k}$ to $-u$.

Lemma 2.3.6. Let $\mathcal{J}$ be elliptic in the sense of Definition 2.2.1 If $\left\{u_{k}\right\} \subset \mathrm{B}\left(\mathbb{R}^{n}\right)$ is a sequence of viscosity subsolutions to $\mathcal{J} u_{k}=f_{k}$ on $\Omega$ such that

(a) $\left\{-u_{k}\right\} \Gamma$-converges to $-u$ in $\Omega,(b)\left\{u_{k}\right\}$ converges to $u$ a.e. on $\mathbb{R}^{n}$ and

(c) $\left\{f_{k}\right\}$ converges to $f$ locally uniformly on $\Omega$, then $u$ is a viscosity subsolution to $\mathcal{J} u=f$ on $\Omega$.

As a corollary, we also obtain the stability property under uniform limits. 
Corollary 2.3.7. Let $\mathcal{J}$ be elliptic in the sense of Definition 2.2.1 If $\left\{u_{k}\right\} \subset \mathrm{B}\left(\mathbb{R}^{n}\right)$ is a sequence of viscosity solutions to $\mathcal{J} u_{k}=f_{k}$ on $\Omega$ such that

(a) $\left\{u_{k}\right\}$ and $\left\{f_{k}\right\}$ converge to $u$ and $f$ locally uniformly on $\Omega$, respectively,

(b) $\left\{u_{k}\right\}$ converges to $u$ a.e. on $\mathbb{R}^{n}$, then $u$ is a viscosity solution to $\mathcal{J} u=f$ on $\Omega$.

Proof. Since $u_{k} \rightarrow u$ locally uniformly on $\Omega$, we see that $\left\{u_{k}\right\} \Gamma$-converges to $u$ in $\Omega$. Thus the required result follows from Lemma 2.3.5 and 2.3.6.

2.4. Comparison principle. The comparison principle for viscosity solutions can be shown by very standard ideas in nonlinear analysis, which originated from the idea of Jensen [J] using sup-convolutions and infconvolutions. The method has been succesfully adapted to integro-differential equations $[\mathrm{A}]$ and a more general proof can be found in $[\overline{\mathrm{BI}}]$ in case that the viscosity solutions have an arbitrary growth at infinity. Our definitions do not quite fit in with the previous frameworks because we consider mainly the general class of operators given by Definition 2.2.1 and we allow discontinuities outside of the domain $\Omega$ of the equation. However the similar techniques can be applied to our equations by the stability property on viscosity subsolutions and supersolutions.

The key result of this section that is crucial for our regularity theory is Theorem 2.4.4, because we can apply it to incremental quotients of viscosity solutions to fully nonlinear integro-differential equations to get its $C^{1, \alpha}$ estimates in Section 5.3.

In order to obtain a comparison principle for a nonlinear operator $\mathcal{J}$, we need to impose a minimal ellipticity condition to our collection $\mathfrak{L}$ of linear operators as follows (see also [CS]).

Assumption 2.4.1. There is a constant $R_{0} \geq 1$ so that for each $R>R_{0}$ and $\sigma \in(1,2)$ there exists some $\delta=\delta(\sigma, R)>0$ such that for any $\mathcal{L} \in \mathfrak{L}$ we have that $\mathcal{L} \varphi>\delta$ on $B_{R^{2-\sigma}}$ where $\varphi$ is a function given by $\varphi(x)=R^{5} \wedge|x|^{2}$.

Assumption 2.4.1 is enough for the comparison principle. We note that Assumption 2.4.1 is very mild. In fact, we shall show in the next lemma that the class $\mathfrak{I}_{0}$ satisfies Assumption 2.4.1. It just says that, given the particular function $R^{5} \wedge|x|^{2}$, the value of the operator will be strictly positive on $B_{R^{2-\sigma}}$ but it does not require any uniform estimate on how that happens.

Assumption 2.4 .1 is pretty mild. Indeed, the following lemma can be shown by simple computation. So we just state it without detailed proof.

Lemma 2.4.2. If $1<\sigma<2$, then $\mathfrak{Q}_{0}=\bigcup_{t \geq 1 / 2} \mathfrak{L}_{t}$ satisfies Assumption 2.4.1.

Theorem 2.4.3. Let $\mathcal{J}$ be elliptic with respect to $\mathfrak{I}$ in the sense of Definition 2.2.1 where $\mathfrak{I}$ is some class satisfying Assumption 2.4.1 and let $\Omega \subset \mathbb{R}^{n}$ be a bounded domain. If $u \in \mathrm{B}\left(\mathbb{R}^{n}\right)$ is a viscosity subsolution to $\mathcal{J} u \geq f$ on $\Omega, v \in \mathrm{B}\left(\mathbb{R}^{n}\right)$ is a viscosity supersolution to $\mathcal{J} v \leq f$ on $\Omega$ and $u \leq v$ on $\mathbb{R}^{n} \backslash \Omega$, then $u \leq v$ on $\Omega$.

We obtain in Theorem 2.4.4 the result which allows functions $u$ and $v$ to be discontinuous on $\mathbb{R}^{n} \backslash \Omega$ and is useful in proving the comparison principle. Its proof can be done by a nonsymmetric adaptation of the proof in [CS]. 
Theorem 2.4.4. Let $\mathcal{J}$ be elliptic with respect to some class $\mathfrak{I}$ in the sense of Definition 2.2.1 and let $\Omega \subset \mathbb{R}^{n}$ be a bounded domain. If $u \in \mathrm{B}\left(\mathbb{R}^{n}\right)$ is a viscosity subsolution to $\mathcal{J} u \geq f$ on $\Omega$ and $v \in \mathrm{B}\left(\mathbb{R}^{n}\right)$ is a viscosity supersolution to $\mathcal{J} v \leq g$ on $\Omega$, then $\mathcal{M}_{\mathfrak{L}}^{+}[u-v] \geq f-g$ on $\Omega$ in the viscosity sense.

Lemma 2.4.5. Let $\mathfrak{L}$ be a class of linear integro-differential operators which satisfies Assumption 2.4.1 If $u \in \mathrm{B}\left(\mathbb{R}^{n}\right)$ is a viscosity subsolution to $\mathcal{M}_{\mathfrak{\Omega}}^{+} u \geq 0$ on $\Omega$, then $\sup _{\Omega} u \leq \sup _{\mathbb{R}^{n} \backslash \Omega} u$.

Proof. Given $\sigma \in(1,2)$, take a sufficiently large $R>0$ so that $\Omega \subset B_{R^{2-\sigma}}$. For $\epsilon>0$ and $M \in \mathbb{R}$, let $\varphi_{M}^{\epsilon}(x)=M+\frac{\epsilon}{R^{5}}\left(R^{5}-R^{5} \wedge|x|^{2}\right)$. Then $M \leq \varphi_{M}^{\epsilon}(x) \leq M+\epsilon$ for any $x \in \mathbb{R}^{n}$. Since $\mathcal{L} 1=0$ for all $\mathcal{L} \in \mathbb{L}$, by Assumption 2.4.1 there is a $\delta>0$ so that $\mathcal{M}_{\mathfrak{\Omega}}^{+}\left[\varphi_{M}^{\epsilon}\right](x) \leq-\epsilon \delta / R^{5}$ for any $x \in B_{R^{2-\sigma}}$. Then we can complete the proof by applying a similar method as in [CS].

[Proof of Theorem 2.4.3] By Theorem 2.4.4, we see that $\mathcal{M}_{\Omega}^{+}[u-v] \geq 0$ on $\Omega$ in the viscosity sense. Applying Lemma 2.4.5, we obtain that $\sup _{\Omega}[u-v] \leq$ $\sup _{\mathbb{R}^{n} \backslash \Omega}[u-v]$. Hence this completes the proof.

Remark 2.4.6. Once we obtain the comparison principle for viscosity subsolutions and supersolutions which is semicontinuous on $\bar{\Omega}$, existence of the solutions of the Dirichlet problem that we mentioned in the introduction follows from the Perron's method [I] as long as we can construct suitable barriers. For a domain $\Omega$ which has the exterior ball condition and prescribed boundary data in $\mathbb{R}^{n} \backslash \Omega$ being continuous, the function $\Psi$ to be constructed in Section 4 can be used as the barriers.

\section{A nonlocal Alexandroff-Bakelman-Pucci estimate}

The Alexandroff-Bakelman-Pucci (A-B-P) estimate plays an important role in Krylov and Sofonov theory [KS], which is an essential tool in the proof of Harnack inequality for linear uniformly elliptic equations with measurable coefficients. In this section, the influence of the gradient term has been addressed in our proof of A-B-P estimate to which converges as $\sigma$ is getting close to 2 . In a later section, we shall use this nonlocal version of the A-B-P estimte to prove Hölder estimates for $\sigma$ close to 2.

Let $u: \mathbb{R}^{n} \rightarrow \mathbb{R}$ be a function which is not positive outside the ball $B_{1 / 2}$ and is upper semicontinuous on $\bar{B}_{1}$. We consider its concave envelope $\Gamma$ in $B_{3}$ defined as $\Gamma(x)=\inf \left\{p(x): p \in \Pi, p>u^{+}\right.$in $\left.B_{2}\right\}$ in $B_{2}$ and 0 in $\mathbb{R}^{n} \backslash B_{2}$, where $\Pi$ is the family of all the hyperplanes in $\mathbb{R}^{n}$. Also we denote the contact set of $u$ and $\Gamma$ in $B_{1}$ by $C\left(u, \Gamma, B_{1}\right)=\left\{y \in B_{1}: u(y)=\Gamma(y)\right\}$.

\subsection{Key Lemma for nonlocal A-B-P estimate.}

Lemma 3.1.1. Let us assume $1<\sigma<2$. Let $u \leq 0$ in $\mathbb{R}^{n} \backslash B_{1 / 2}$ and let $\Gamma$ be its concave envelope in $B_{2}$. If $u \in \mathrm{B}\left(\mathbb{R}^{n}\right)$ is a viscosity subsolution to $\mathcal{M}_{\mathfrak{L}_{0}}^{+} u=-f$ on $B_{1}$ where $f: \mathbb{R}^{n} \rightarrow \mathbb{R}$ is a function with $f>0$ on $C\left(u, \Gamma, B_{1}\right)$, then there exists some constant $C>0$ depending only on $n, \lambda$ and $\Lambda$ (but not on $\sigma$ ) such that for 
any $x \in C\left(u, \Gamma, B_{1}\right)$ and any $M>0$ there is some $k \in \mathbb{N} \cup\{0\}$ such that

$$
\left|\left\{y \in R_{k}(x): \underline{\mu}^{-}(u, x, y ; \nabla \Gamma) \geq M r_{k}^{2}\right\}\right| \leq C \frac{f(x)+|\nabla \Gamma(x)|}{M}\left|R_{k}(x)\right|
$$

where $R_{k}(x)=B_{r_{k}}(x) \backslash B_{r_{k+1}}(x)$ for $r_{k}=\varrho_{0} 2^{-\frac{1}{2-\sigma}-k}$ and $\varrho_{0}=1 /(16 \sqrt{n})$. Here, $\nabla \Gamma(x)$ denotes any element of the superdifferential $\partial \Gamma(x)$ of $\Gamma$ at $x$.

Remark. We note that $\nabla \Gamma(x)=\nabla u(x)$ for $x \in B_{1}$ if $\Gamma$ and $u$ are differentiable at $x \in B_{1}$. In this case, $\partial \Gamma(x)$ is a singleton set with element $\nabla u(x)$.

[Proof of Lemma 3.1.1] Take any $x \in C\left(u, \Gamma, B_{1}\right)$. Since $u$ can be touched by a hyperplane from above at $x$, we see $\nabla \varphi(x)=\nabla \Gamma(x)$ for a $\varphi \in C_{\Omega}^{2}(u ; x)^{+}$. Thus it follows from Theorem 2.2.4 that $\mathcal{M}_{\mathfrak{S}_{0}}^{+} u(x ; \nabla \Gamma)$ is well-defined. We observe that $\mu_{t}(u, x, y ; \nabla \Gamma)=u(x+y)-u(x)-(\nabla \Gamma(x) \cdot y) \chi_{B_{t}}(y) \leq 0$ for any $y \in B_{1 / 2}$ and $t \geq 1 / 2$, by the definition of concave envelope of $u$ in $B_{2}$. Since $\mu_{t}^{+}(u, x, y ; \nabla \Gamma) \leq|\nabla \Gamma(x)||y|$ for any $t \geq 1 / 2$, we obtain that

$$
\sup _{t \geq 1 / 2} \int_{\mathbb{R}^{n}} \frac{\Lambda \mu_{t}^{+}(u, x, y ; \nabla \Gamma)}{|y|^{n+\sigma}} d y \leq \int_{\mathbb{R}_{n} \backslash B_{1 / 2}} \frac{\Lambda|\nabla \Gamma(x)||y|}{|y|^{n+\sigma}} d y \fallingdotseq c_{0}|\nabla \Gamma(x)| .
$$

Then the constant $c_{0}>0$ depending only on $n, \sigma$ and $\Lambda$ is finite for $\sigma>1$. Thus by Theorem 2.2.4 we have that

$$
\begin{aligned}
& -f(x) \leq \mathcal{M}_{\mathbb{S}_{0}}^{+} u(x ; \nabla \Gamma) \\
& =(2-\sigma) \sup _{t \geq 1 / 2} \int_{\mathbb{R}^{n}} \frac{\Lambda \mu_{t}^{+}(u, x, y ; \nabla \Gamma)-\lambda \mu_{t}^{-}(u, x, y ; \nabla \Gamma)}{|y|^{n+\sigma}} d y \\
& \leq(2-\sigma) \sup _{t \geq 1 / 2} \int_{\mathbb{R}^{n}} \frac{\Lambda \mu_{t}^{+}(u, x, y ; \nabla \Gamma)}{|y|^{n+\sigma}} d y-(2-\sigma) \inf _{t \geq 1 / 2} \int_{\mathbb{R}^{n}} \frac{\lambda \mu_{t}^{-}(u, x, y ; \nabla \Gamma)}{|y|^{n+\sigma}} d y \\
& \leq(2-\sigma) \int_{\mathbb{R}_{n} \backslash B_{1 / 2}} \frac{\Lambda|\nabla \Gamma||y|}{|y|^{n+\sigma}} d y-(2-\sigma) \int_{\mathbb{R}^{n}} \frac{\lambda \mu^{-}(u, x, y ; \nabla \Gamma)}{|y|^{n+\sigma}} d y \\
& \leq(2-\sigma) \mathcal{C}_{0}|\nabla \Gamma(x)|-(2-\sigma) \int_{B_{r_{0}}(x)} \frac{\lambda \mu_{1}^{-}(u, x, y ; \nabla \Gamma)}{|y|^{n+\sigma}} d y,
\end{aligned}
$$

where $r_{0}=\varrho_{0} 2^{-\frac{1}{2-\sigma}}$. Splitting the above integral in the rings $R_{k}(x)$, we have that

$$
f(x) \geq(2-\sigma) \lambda \sum_{k=0}^{\infty} \int_{R_{k}(x)} \frac{\mu_{1}^{-}(u, x, y ; \nabla \Gamma)}{|y|^{n+\sigma}} d y-(2-\sigma) c_{0}|\nabla \Gamma(x)| .
$$

Assume that the conclusion (3.1.1) does not hold, i.e. for any $C>0$ there are some $x_{0} \in C\left(u, \Gamma, B_{1}\right)$ and $M_{0}>0$ such that

$$
\left|\left\{y \in R_{k}\left(x_{0}\right): \underline{\mu}^{-}\left(u, x_{0}, y ; \nabla \Gamma\right) \geq M_{0} r_{k}^{2}\right\}\right|>C \frac{f\left(x_{0}\right)+\left|\nabla \Gamma\left(x_{0}\right)\right|}{M_{0}}\left|R_{k}\left(x_{0}\right)\right|
$$


for all $k \in \mathbb{N} \cup\{0\}$. Since $-\mu_{1} \leq \mu_{1}^{-}$and $(2-\sigma) \frac{1}{1-2^{-(2-\sigma)}}$ remains bounded below for $\sigma \in(1,2)$, it follows from (3.1.2) that

$$
\begin{aligned}
f\left(x_{0}\right)+(2-\sigma) c_{0}\left|\nabla \Gamma\left(x_{0}\right)\right| & \geq(2-\sigma) \lambda \sum_{k=0}^{\infty} \int_{R_{k}\left(x_{0}\right)} \frac{-\mu_{1}\left(u, x_{0}, y ; \nabla \Gamma\right)}{|y|^{n+\sigma}} d y \\
& \geq c(2-\sigma) \sum_{k=0}^{\infty} M_{0} \frac{r_{k}^{2}}{r_{k}^{\sigma}} C \frac{f\left(x_{0}\right)+\left|\nabla \Gamma\left(x_{0}\right)\right|}{M_{0}} \\
& \geq \frac{c C(2-\sigma) \rho_{0}^{2}}{1-2^{-(2-\sigma)}}\left(f\left(x_{0}\right)+\left|\nabla \Gamma\left(x_{0}\right)\right|\right) \\
& \geq c C\left(f\left(x_{0}\right)+\left|\nabla \Gamma\left(x_{0}\right)\right|\right)
\end{aligned}
$$

for any $C>0$. Taking $C$ large enough, we obtain a contradiction. Hence we are done.

Remark. Lemma 3.1 .1 would hold for any particular choice of $\varrho_{0}$ by modifying $C$ accordingly. The particular choice $\varrho_{0}=1 /(16 \sqrt{n})$ is convenient for the proofs in Section 4

Lemma 3.1.2. [CS] Let $\Gamma$ be a concave function on $B_{r}(x)$ where $x \in \mathbb{R}^{n}$ and let $h>0$. If $\left|\left\{y \in S_{r}(x): \Gamma(y)<\Gamma(x)+(y-x) \cdot \nabla \Gamma(x)-h\right\}\right| \leq \epsilon\left|S_{r}(x)\right|$ for any small $\epsilon>0$ where $S_{r}(x)=B_{r}(x) \backslash B_{r / 2}(x)$, then we have $\Gamma(y) \geq \Gamma(x)+(y-x) \cdot \nabla \Gamma(x)-h$ for any $y \in B_{r / 2}(x)$.

Corollary 3.1.3. For any $\epsilon>0$, there is a constant $C>0$ such that for any function $u$ with the same hypothesis as Lemma 3.1.1 there is some $r \in\left(0, \varrho_{0} 2^{-\frac{1}{2-\sigma}}\right)$ such that

$$
\begin{aligned}
& \frac{\left|\left\{y \in S_{r}(x): u(y)<u(x)+(y-x) \cdot \nabla \Gamma(x)-C(f(x)+|\nabla \Gamma(x)|) r^{2}\right\}\right|}{\left|S_{r}(x)\right|} \leq \epsilon, \\
& \int_{\bar{Q}} g_{\eta}(\nabla \Gamma(y)) \operatorname{det}\left[D^{2} \Gamma(y)\right]^{-} d y \leq C \sup _{y \in \bar{Q}}\left(1+\eta^{-n}|f(y)|^{n}\right)|Q|
\end{aligned}
$$

for any $\eta>0$ and any cube $Q \subset B_{r / 4}(x)$ with diameter $d$ such that $x \in \bar{Q}$ and $r / 4<d<r / 2$, where $\varrho_{0}=1 /(16 \sqrt{n})$ and $g_{\eta}(z)=\left(|z|^{n /(n-1)}+\eta^{n /(n-1)}\right)^{1-n}$.

Proof. The first part can be obtained by choosing $M=C(f(x)+|\nabla \Gamma(x)|) / \epsilon$ in Lemma 3.1.1. Also the second part follows as a consequence of Lemma 3.1.2 and concavity;

$$
\operatorname{det}\left[D^{2} \Gamma(x)\right]^{-} \leq C(f(x)+|\nabla \Gamma(x)|)^{n} \leq 4^{n} C \frac{\left(1+\eta^{-n}|f(x)|^{n}\right)}{g_{\eta}(\nabla \Gamma(x))} .
$$

Thus we have that $g_{\eta}(\nabla \Gamma(x)) \operatorname{det}\left[D^{2} \Gamma(x)\right]^{-} \leq 4^{n} C\left(1+\eta^{-n}|f(x)|^{n}\right)$.

Take any $y \in C\left(u, \Gamma, B_{1}\right) \cap Q$ where $Q \subset B_{r / 4}(x)$ is a cube with diameter $d$ such that $x \in \bar{Q}$ and $r / 4<d<r / 2$. Similarly to the above, we can obtain that $g_{\eta}(\nabla \Gamma(\cdot)) \operatorname{det}\left[D^{2} \Gamma(\cdot)\right]^{-} \leq 4^{n} C\left(1+\eta^{-n}|f(\cdot)|^{n}\right)$ a.e. on $Q$ because $\operatorname{det}\left[D^{2} \Gamma(\cdot)\right]^{-}=0$ a.e. on $Q \backslash C\left(u, \Gamma, B_{1}\right)$ as in [CC]. Hence this implies the second part. 
3.2. A nonlocal A-B-P estimate. We obtain a nonlocal version of AlexandroffBakelman-Pucci estimate in the following theorem.

Theorem 3.2.1. Let $u$ and $\Gamma$ be functions as in Lemma 3.1.1 Then there exist a finite family $\left\{Q_{j}\right\}_{j=1}^{m}$ of open cubes $Q_{j}$ with diameters $d_{j}$ such that

(a) any two cubes $Q_{i}$ and $Q_{j}$ do not intersect, $(b) C\left(u, \Gamma, B_{1}\right) \subset \bigcup_{j=1}^{m} \bar{Q}_{j}$,

(c) $C\left(u, \Gamma, B_{1}\right) \cap \bar{Q}_{j} \neq \phi$ for any $Q_{j},(d) d_{j} \leq \varrho_{0} 2^{-\frac{1}{2-\sigma}}$ where $\varrho_{0}=1 /(16 \sqrt{n})$,

(e) $\int_{\bar{Q}_{j}} g_{\eta}(\nabla \Gamma(y)) \operatorname{det}\left[D^{2} \Gamma(y)\right]^{-} d y \leq C \sup _{\bar{Q}_{j}}\left(1+\eta^{-n}|f|^{n}\right)\left|Q_{j}\right|$,

(f) $\left|\left\{y \in 4 \sqrt{n} Q_{j}: u(y) \geq \Gamma(y)-C\left(\sup _{\bar{Q}_{j}}(f+|\nabla \Gamma|)\right) d_{j}^{2}\right\}\right| \geq \gamma\left|Q_{j}\right|$,

where the constants $C>0$ and $\gamma>0$ depend on $n, \Lambda$ and $\lambda$ (but not on $\sigma$ ).

Proof. In order to obtain such a family, we start by covering $B_{1}$ with a tiling of cubes of diameter $\varrho_{0} 2^{-\frac{1}{2-\sigma}}$. Then discard all those that do not intersect $C\left(u, \Gamma, B_{1}\right)$. Whenever a cube does not satisfy (e) and (f), we split it into $2^{n}$ cubes of half diameter and discard those whose closure does not intersect $C\left(u, \Gamma, B_{1}\right)$. Now our goal is to prove that eventually all cubes satisfy (e) and (f) and this process ends after a finite number of steps.

Assume that the process does not finish in a finite number of steps. Then we can have an infinite nested sequence of cubes. The intersection of their closures will be a point $\hat{x}$. So we may choose a sequence $\left\{x_{k}\right\} \subset$ $C\left(u, \Gamma, B_{1}\right)$ with $\lim _{k \rightarrow \infty} x_{k}=\hat{x}$. Since $u\left(x_{k}\right)=\Gamma\left(x_{k}\right)$ for all $k \in \mathbb{N}$, by the upper semicontinuity of $u$ on $\bar{B}_{1}$ we have that $\Gamma(\hat{x})=\limsup _{k \rightarrow \infty} u\left(x_{k}\right) \leq u(\hat{x})$. Also we have that $u(\hat{x}) \leq \Gamma(\hat{x})$ because $u \leq \Gamma$ on $B_{2}$ by the definition of the concave envelope $\Gamma$ in $B_{3}$. Thus we obtain that $u(\hat{x})=\Gamma(\hat{x})$. We will now get a contradiction by showing that eventually one of these cubes containing $\hat{x}$ will not split.

Take any $\epsilon>0$. By Corollary 3.1 .3 there is some $r \in\left(0, \varrho_{0} 2^{-\frac{1}{2-\sigma}}\right)$ such that

$$
\begin{aligned}
& \frac{\left|\left\{y \in S_{r}(\hat{x}): u(y)<u(\hat{x})+(y-\hat{x}) \cdot \nabla \Gamma(\hat{x})-C(f(\hat{x})+|\nabla \Gamma(\hat{x})|) r^{2}\right\}\right|}{\left|S_{r}(\hat{x})\right|} \leq \epsilon, \\
& \int_{\bar{Q}_{j}} g_{\eta}(\nabla \Gamma(y)) \operatorname{det}\left[D^{2} \Gamma(y)\right]^{-} d y \leq C \sup _{y \in \bar{Q}_{j}}\left(1+\eta^{-n}|f(y)|^{n}\right)\left|Q_{j}\right|
\end{aligned}
$$

for any $\eta>0$ and a cube $Q_{j} \subset B_{r / 4}(x)$ with diameter $d_{j}$ such that $x \in \bar{Q}_{j}$ and $r / 4<d_{j}<r / 2$. So we easily see that $\bar{Q}_{j} \subset B_{r / 2}(\hat{x})$ and $B_{r}(\hat{x}) \subset 4 \sqrt{n} Q_{j}$. We recall that $\Gamma(y) \leq u(\hat{x})+(y-\hat{x}) \cdot \nabla \Gamma(\hat{x})$ for any $y \in B_{2}$ because $\Gamma$ is concave on $B_{2}$ and $\Gamma(\hat{x})=u(\hat{x})$. Since $d_{j}$ is comparable to $r$, it thus follows that

$$
\begin{aligned}
\mid\{y \in & \left.4 \sqrt{n} Q_{j}: u(y) \geq \Gamma(y)-C\left(\sup _{\bar{Q}_{j}}(f+\nabla \Gamma)\right) d_{j}^{2}\right\} \mid \\
& \geq\left|\left\{y \in 4 \sqrt{n} Q_{j}: u(y) \geq u(\hat{x})+(y-\hat{x}) \cdot \nabla \Gamma(\hat{x})-C(f(\hat{x})+\nabla \Gamma(\hat{x})) r^{2}\right\}\right| \\
& \geq(1-\epsilon)\left|S_{r}(\hat{x})\right| \geq \gamma\left|Q_{j}\right| .
\end{aligned}
$$


Thus we proved (f). Moreover, (e) holds for $Q_{j}$ because $\bar{Q}_{j} \subset B_{r / 2}(\hat{x})$ and $B_{r}(\hat{x}) \subset 4 \sqrt{n} Q_{j}$. Hence the cube $Q_{j}$ would not split and the process must stop there.

Remark 3.2.2. Note that the upper bound for the diameters $\varrho_{0} 2^{-\frac{1}{2-\sigma}}$ becomes very small as $\sigma$ is getting close to 2 . Adding $\sum_{j=1}^{m}\left|\nabla \Gamma\left(Q_{j}\right)\right|$ and taking $\sigma \rightarrow 2$, we obtain the classical Alexandroff estimate as the limit of the Riemann sums. For each $\sigma>0$, we have that

$$
\int_{C\left(u, \Gamma, B_{1}\right)} g_{\eta}(\nabla \Gamma(y)) \operatorname{det}\left[D^{2} \Gamma(y)\right]^{-} d y \leq C \sum_{j} \sup _{y \in \bar{Q}_{j}}\left(1+\eta^{-n}|f(y)|^{n}\right)\left|Q_{j}\right| .
$$

As $\sigma \rightarrow 2$, the cube covering of $C\left(u, \Gamma, B_{1}\right)$ is getting close to the contact set $C\left(u, \Gamma, B_{1}\right)$ and the above becomes the following estimate (refer to Ch. 9.1 of [GT]);

$$
\int_{B_{M_{0}}} g_{\eta}(z) d z \leq C \int_{C\left(u, \Gamma, B_{1}\right)}\left(1+\eta^{-n}|f(y)|^{n}\right) d y
$$

for any $\eta>0$, where $M_{0}=\sup _{B_{1}} u^{+}$. Since $g_{\eta}(z) \geq 2^{2-n}\left(|z|^{n}+\eta^{n}\right)^{-1}$, we have

$$
\ln \left(\frac{M_{0}^{n}}{\eta^{n}}+1\right) \leq C\left(\left|B_{1}\right|+\eta^{-n}\|f\|_{L^{n}\left(C\left(u, \Gamma, B_{1}\right)\right)}^{n}\right)
$$

If we set $\eta=\|f\|_{L^{n}\left(C\left(u, \Gamma, B_{1}\right)\right)}$, then we obtain that $\sup _{B_{1}} u^{+} \leq C\|f\|_{L^{n}\left(C\left(u, \Gamma, B_{1}\right)\right)}$.

\section{Decay estimates of UPper LeVel Sets}

In this section, we are going to apply the A-B-P estimate to get the geometric decay rate of upper level sets for a nonnegative subsolution $u$. To do this, we need a special function $\Psi$ so that $\Psi-u$ meets the conditions of the A-B-P estimate. It is based on the method used in [CS], but nontrivial computations have been done to create positive terms so that it absorbs the influence of the gradient term.

\subsection{Special Functions.}

Lemma 4.1.1. There exist some $\sigma^{*} \in(1,2)$ and $p>0$ such that the function $f(x)=2^{p} \wedge|x|^{-p}$ is a subsolution to $\mathcal{M}_{\mathfrak{Q}_{0}}^{-} f(x) \geq 0$ for any $\sigma \in\left(\sigma^{*}, 2\right)$ and $x \in B_{1}^{c}$.

Proof. It is enough to show that there is some $\sigma^{*} \in(1,2)$ so that

$$
\mathcal{M}_{\mathfrak{L}_{0}}^{-} f(x) \geq 0
$$

for $x=e_{n}=(0, \cdots, 0,1) \in \mathbb{R}^{n}$; for every other $x$ with $|x|=1$, the above inequality follows by rotation. If $|x| \geq 1$, then we consider $g(y)=|x|^{p} f(|x| y)$. Note that $g(y)=2^{p}|x|^{p}$ for $|y|<1 /(2|x|)$ and $|y|^{-p}$ for $|y| \geq 1 /(2|x|)$. Then we can derive that $g(y) \geq f(y)$ for any $y \in \mathbb{R}^{n}, f(x /|x|)=g(x /|x|), \nabla f(x /|x|)=$ $\nabla g(x /|x|)$ and

$$
\nabla f\left(\frac{x}{|x|}\right) \cdot y=|x|^{p}(\nabla f(x) \cdot(|x| y))
$$


for $y \in B_{1 / 2}$. Thus we see that $\mu_{t}(g, x /|x|, y) \geq \mu_{t}(f, x /|x|, y)$ for any $t \geq 1 / 2$. We denote by $\mathcal{K}_{0}$ the class of all positive kernels satisfying (2.1.3) and (2.1.4). For $t \geq 1 / 2$ and $K \in \mathcal{K}_{0}$, we define the map $K_{t}$ given by $K_{t}(y)=t^{-n-\sigma} K(y / t)$. Then it is easy to check that the mapping $\mathcal{K}_{0} \rightarrow \mathcal{K}_{0}$ given by $K \mapsto K_{|x|}$ is an isometry because $K \mapsto K_{1 /|x|}$ is its inverse mapping. Since $|\nabla f(x)|=p /|x|^{p+1}$, it follows from (4.1.2) and the change of variables that

$$
\begin{aligned}
\mathcal{M}_{\mathfrak{I}_{0}}^{-} g(x /|x|) & \leq \mathcal{L}^{t} g(x /|x|) \\
& =|x|^{\sigma+p} \int_{\mathbb{R}^{n}}\left[f(x+y)-f(x)-(\nabla f(x) \cdot y) \chi_{B_{t|x|}}(y)\right] K_{|x|}(y) d y \\
& \fallingdotseq|x|^{\sigma+p} \mathcal{L}_{|x|}^{t|x|} f(x)
\end{aligned}
$$

for any $\mathcal{L}^{t} \in \mathfrak{L}_{0}$ and $\sigma \in\left(\sigma^{*}, 2\right)$. Since $t|x| \geq 1 / 2$ and the mapping $\mathcal{K}_{0} \rightarrow \mathcal{K}_{0}$ given by $K \mapsto K_{|x|}$ is an isometry, by taking the infimum of both sides on $\mathfrak{L}_{0}$ in the above inequality we obtain that

$$
\mathcal{M}_{\mathfrak{\Omega}_{0}}^{-} g(x /|x|) \leq|x|^{\sigma+p} \mathcal{M}_{\mathfrak{Q}_{0}}^{-} f(x)
$$

for any $\sigma \in\left(\sigma^{*}, 2\right)$. By (4.1.1), (4.1.2) and (4.1.3), we conclude that

$$
\mathcal{M}_{\mathfrak{Q}_{0}}^{-} f(x) \geq \frac{1}{|x|^{\sigma+p}} \mathcal{M}_{\mathfrak{Q}_{0}}^{-} g(x /|x|) \geq \frac{1}{|x|^{\sigma+p}} \mathcal{M}_{\mathfrak{Q}_{0}}^{-} f(x /|x|) \geq 0
$$

for any $\sigma \in\left(\sigma^{*}, 2\right)$.

In order to prove (4.1.1), we use the following elementary inequality that holds for any $a>b>0$ and $p>0$;

$$
(a+b)^{-p} \geq a^{-p}\left(1-p \frac{b}{a}+\frac{p(p+1)}{2 !}\left(\frac{b}{a}\right)^{2}-\frac{p(p+1)(p+2)}{3 !}\left(\frac{b}{a}\right)^{3}\right) .
$$

Using this inequality, we have that

$$
\begin{aligned}
\mu_{t}\left(f, e_{n}, y\right) & =\left|e_{n}+y\right|^{-p}-1+p y_{n}=\left(1+|y|^{2}+2 y_{n}\right)^{-p / 2}-1+p y_{n} \\
& \geq-\left(\frac{p}{2}+1\right)|y|^{2}+\frac{p(p+2)}{2} y_{n}|y|^{2}+\frac{|y|^{2}}{\left(1+|y|^{2}\right)^{p / 2+1}} \\
& +\frac{p(p+2)}{2} \frac{y_{n}^{2}}{\left(1+|y|^{2}\right)^{p / 2+2}}-\frac{p(p+2)(p+4)}{6} \frac{y_{n}^{3}}{\left(1+|y|^{2}\right)^{p / 2+3}}
\end{aligned}
$$

for any $t \geq 1 / 2$ and $y \in B_{1 / 2}$. We now choose some sufficiently large $p>0$ so that

$$
\frac{p(p+2)}{2\left(1+r^{2}\right)^{p / 2+2}} \int_{S^{n-1}} \theta_{n}^{2} d \sigma(\theta)+\frac{\omega_{n}}{\left(1+r^{2}\right)^{p / 2+1}}-\left(\frac{p}{2}+1\right) \omega_{n}=\delta_{0}(r)>0
$$

for any sufficiently small $r>0$, where $\omega_{n}$ is the surface measure of $S^{n-1}$. Since $\int_{S^{n-1}} \theta_{n} d \sigma(\theta)=\int_{S^{n-1}} \theta_{n}^{3} d \sigma(\theta)=0$ and $\mu_{t}^{-}\left(f, e_{n}, y\right) \leq 2^{p}+1+p$ for any 
$t \geq 1 / 2$ and $y \in B_{1 / 2}$, it follows from (2.2.1), (2.2.2), (4.1.4) and 4.1.5) that

$$
\begin{aligned}
& \mathcal{M}_{\mathfrak{I}_{0}}^{-} f\left(e_{n}\right) \\
& \geq(2-\sigma) \inf _{t \geq 1 / 2} \int_{\mathbb{R}^{n}} \frac{\lambda \mu_{t}^{+}\left(f, e_{n}, y\right)}{|y|^{n+\sigma}} d y-(2-\sigma) \sup _{t \geq 1 / 2} \int_{\mathbb{R}^{n}} \frac{\Lambda \mu_{t}^{-}\left(f, e_{n}, y\right)}{|y|^{n+\sigma}} d y \\
& \geq(2-\sigma) \lambda \inf _{t \geq 1 / 2} \int_{B_{r}} \frac{\mu_{t}\left(f, e_{n}, y\right)}{|y|^{n+\sigma}} d y-(2-\sigma) \Lambda \sup _{t \geq 1 / 2} \int_{\mathbb{R}^{n} \backslash B_{r}} \frac{\mu_{t}^{-}\left(f, e_{n}, y\right)}{|y|^{n+\sigma}} d y \\
& \geq(2-\sigma)\left(\frac{\lambda \omega_{n} \delta_{0}(r)}{2-\sigma}-\left(2^{p}+1+p\right) \Lambda \int_{\mathbb{R}^{n} \backslash B_{r}} \frac{1}{|y|^{n+\sigma}} d y\right) \\
& =\lambda \omega_{n} \delta_{0}(r)-\left(2^{p}+1+p\right) \Lambda \omega_{n} \frac{2-\sigma}{\sigma} r^{-\sigma}
\end{aligned}
$$

for $r \in(0,1 / 2)$. Thus we may take some sufficiently small $r \in(0,1 / 2)$ and take some $\sigma^{*} \in(1,2)$ close enough to 2 in the above so that $\mathcal{M}_{\mathfrak{I}_{0}}^{-} f\left(e_{n}\right) \geq 0$ for any $\sigma \in\left(\sigma^{*}, 2\right)$. Hence we complete the proof.

Corollary 4.1.2. Given any $\sigma_{0} \in(1,2)$, there exist some $\delta>0$ and $p>n$ such that the function $f_{\delta}(x)=\delta^{-p} \wedge|x|^{-p}$ is a subsolution to $\mathcal{M}_{\mathfrak{I}_{0}}^{-} f_{\delta}(x) \geq 0$ for any $\sigma \in\left(\sigma_{0}, 2\right), x \in B_{1}^{c}$.

Proof. Let $\sigma^{*} \in(1,2)$ be the number of Lemma 4.1.1. Without loss of generality, we may assume that $\sigma_{0}<\sigma^{*}$. Lemma4.1.1 implies that the result of this corollary always holds for $\sigma \in\left(\sigma^{*}, 2\right)$, when $\delta=1 / 2$. If $\delta<1 / 2$, then the result still holds for $\sigma \in\left(\sigma^{*}, 2\right)$ because $\mu_{t}\left(f_{\delta}, x, y\right) \geq \mu_{t}\left(f_{1 / 2}, x, y\right)$ for any $y \in \mathbb{R}^{n}, x \in B_{1}^{c}$ and $t \geq 1 / 2$. We shall select $\delta \in(0,1-1 / n)$ so small that the result holds also for $\sigma \in\left(\sigma_{0}, \sigma^{*}\right]$.

Now we let $x=e_{n}$ as in the proof of Lemma 4.1.1. Assume $\sigma_{0}<\sigma \leq \sigma^{*}$. Then we write

$$
\begin{aligned}
\mathcal{M}_{\mathfrak{I}_{0}}^{-} f_{\delta}\left(e_{n}\right) & =(2-\sigma) \lambda \int_{\mathbb{R}^{n}} \frac{\underline{\mu}^{+}\left(f_{\delta}, e_{n}, y\right)}{|y|^{n+\sigma}} d y-(2-\sigma) \Lambda \int_{\mathbb{R}^{n}} \frac{\bar{\mu}^{-}\left(f_{\delta}, e_{n}, y\right)}{|y|^{n+\sigma}} d y \\
& \fallingdotseq \mathcal{J}_{1} f_{\delta}\left(e_{n}\right)+\mathcal{J}_{2} f_{\delta}\left(e_{n}\right) .
\end{aligned}
$$

If we take some $\delta \in(0,1-1 / n)$ small enough so that $\mu_{t}^{-}\left(f_{\delta}, e_{n}, y\right)=0$ for any $y \in B_{3 / 2}$ and $t \geq 1 / 2$, from simple geometric observation it is easy to check that $\mu_{t}^{-}\left(f_{\delta}, e_{n}, y\right) \leq 2^{p}+1+p|y|$ for any $y \in B_{1}^{c}$ and $t \geq 1 / 2$. So we see that

$$
-\mathcal{J}_{2} f_{\delta}\left(e_{n}\right)=(2-\sigma) \Lambda \int_{|y| \geq 1} \frac{\bar{\mu}^{-}\left(f_{\delta}, e_{n}, y\right)}{|y|^{n+\sigma}} d y \leq\left(2-\sigma_{0}\right) \Lambda \int_{|y| \geq 1} \frac{2^{p}+1+p|y|}{|y|^{n+\sigma_{0}}} d y .
$$

Since $\sigma_{0}>1$, we have that $\mathcal{J}_{2} f_{\delta}\left(e_{n}\right) \geq-c_{0}$ for a constant $c_{0}>0$ depending on $\sigma_{0}, \Lambda$ and the dimension $n$. On the other hand, since $|y|<1+\delta$ and 
$-1+p y_{n} \geq-1+n(1-\delta)$ for any $y$ with $\delta / 2<\left|y+e_{n}\right|<\delta$, we have that

$$
\begin{aligned}
\mathcal{J}_{1} f_{\delta}\left(e_{n}\right) & \geq(2-\sigma) \lambda \int_{\delta / 2<\left|y+e_{n}\right|<\delta} \frac{\left|e_{n}+y\right|^{-p}-1+n(1-\delta)}{|y|^{n+\sigma}} d y \\
& \geq \frac{(2-\sigma) \lambda}{(1+\delta)^{n+\sigma}}\left(\int_{\delta / 2<\left|y+e_{n}\right|<\delta}\left|e_{n}+y\right|^{-p} d y+(n-1-\delta n) \delta^{n}\left|B_{1}\right|\left(1-2^{-n}\right)\right) \\
& \geq \frac{(2-\sigma) \lambda}{(1+\delta)^{n+\sigma}} \frac{\omega_{n}}{p-n} \delta^{n-p}\left(2^{p-n}-1\right) .
\end{aligned}
$$

If we select some $\delta \in(0,1-1 / n)$ sufficiently small so that $\mathcal{J}_{1} f_{\delta}\left(e_{n}\right)>c_{0}$, then we can complete the proof.

Lemma 4.1.3. Given any $\sigma_{0} \in(1,2)$, there exists a function $\Psi \in \mathrm{B}\left(\mathbb{R}^{n}\right)$ such that

(a) $\Psi$ is continuous on $\mathbb{R}^{n},(b) \Psi=0$ on $B_{\sqrt{n}}^{c}$,

(c) $\Psi>2$ on $Q_{1},(d) \mathcal{M}_{\mathfrak{Q}_{0}}^{-} \Psi$ is continuous on $B_{\sqrt{n}}$,

(e) $\mathcal{M}_{\mathfrak{Q}_{0}}^{-} \Psi>-\psi$ on $\mathbb{R}^{n}$ where $\psi$ is a positive bounded function on $\mathbb{R}^{n}$ which is supported in $\bar{B}_{1 / 4}$, for any $\sigma \in\left(\sigma_{0}, 2\right)$.

Proof. Let $\delta$ be the number of Corollary 4.1.2. We consider the function $\Psi$ given by $\Psi=0$ in $\mathbb{R}^{n} \backslash B_{\sqrt{n}}, c\left(|x|^{-p}-(\sqrt{n})^{-p}\right)$ in $B_{\sqrt{n}} \backslash B_{\delta}$, and $c P$ in $B_{\delta}$. where $P$ is a quadratic paraboloid chosen so that $\Psi$ is $C^{1,1}$ across $\partial B_{\delta}$. We now choose the constant $c$ so that $\Psi(x)>2$ for $x \in Q_{1}$ (recall that $Q_{1} \subset Q_{2} \subset B_{\sqrt{n}} \subset B_{2 \sqrt{n}}$ ). Since $\Psi \in C^{1,1}\left(B_{\sqrt{n}}\right), \mathcal{M}_{\mathfrak{Q}_{0}}^{-} \Psi$ is continuous on $B_{\sqrt{n}}$. Also by Corollary 4.1.2 we see that $\mathcal{M}_{\mathfrak{I}_{0}}^{-} \Psi \geq 0$ on $B_{1 / 4}^{c}$. Hence this completes the proof.

4.2. Key Lemma. The main tool that shall be useful in proving Hölder estimates is a lemma that connects a pointwise estimate with an estimate in measure. The corresponding lemma in our context is the following.

Lemma 4.2.1. Let $\sigma_{0} \in(1,2)$ be given. If $\sigma \in\left(\sigma_{0}, 2\right)$, then there exist some constants $\varepsilon_{0}>0, v \in(0,1)$ and $M>1$ (depending only on $\sigma_{0}, \lambda, \Lambda$ and the dimension $n$ ) for which if $u \in \mathrm{B}\left(\mathbb{R}^{n}\right)$ is a viscosity supersolution to $\mathcal{M}_{\mathfrak{\Omega}_{0}}^{-} u \leq \varepsilon_{0}$ on $B_{2 \sqrt{n}}$ such that $u \geq 0$ on $\mathbb{R}^{n}$ and $\inf _{Q_{1}} u \leq 1$, then $\left|\{u \leq M\} \cap Q_{1}\right| \geq v$.

Remark. (a) We denote by $Q_{r}(x)$ an open cube $\left\{y \in \mathbb{R}^{n}:|y-x|_{\infty} \leq r / 2\right\}$ and $Q_{r}=Q_{r}(0)$. If we set $Q=Q_{r}(x)$, then we denote by $s Q=Q_{s r}(x)$ for $s>0$.

(b) If we assume that $0<\sigma \leq \sigma^{*}<2$, then there is a simpler proof of Lemma 4.1.3 using the ideas from [S]. The result here is more complicated as in [CS] because we want to get an estimate that remains uniform as $\sigma \rightarrow 2$.

[Proof of Lemma 4.2.1] We consider the function $v \fallingdotseq \Psi-u$ where $\Psi$ is the special function constructed in Lemma 4.1.3. Then we easily see that $v$ is upper semicontinuous on $\bar{B}_{2 \sqrt{n}}$ and $v$ is not positive on $\mathbb{R}^{n} \backslash B_{\sqrt{n}}$. Moreover, $v$ is a viscosity subsolution to $\mathcal{M}_{\mathfrak{Q}_{0}}^{+} v \geq \mathcal{M}_{\mathfrak{Q}_{0}}^{-} \Psi-\mathcal{M}_{\mathfrak{Q}_{0}}^{-} u \geq-\psi-\varepsilon_{0}$ on $B_{2 \sqrt{n}}$. So we want to apply Theorem 3.2.1 (rescaled) to $v$. Let $\Gamma$ be the concave envelope of $v$ in $B_{4 \sqrt{n}}$. 
Since $\inf _{Q_{1}} u \leq 1, \inf _{Q_{1}} \Psi>2$ and $Q_{1} \subset B_{2 \sqrt{n}}$, we easily see that $M_{0} \fallingdotseq$ $\sup _{B_{2 \sqrt{n}}} v=v\left(x_{0}\right)>0$ for some $x_{0} \in B_{2 \sqrt{n}}$. We consider the function $g$ whose graph is the cone in $\mathbb{R}^{n} \times \mathbb{R}$ with vertex $\left(x_{0}, M_{0}\right)$ and base $\partial B_{6 \sqrt{n}}\left(x_{0}\right) \times\{0\}$. For any $\xi \in \mathbb{R}^{n}$ with $|\xi|<M_{0} / 6 \sqrt{n}$, the hyperplane

$$
H=\left\{\left(x, x_{n+1}\right) \in \mathbb{R}^{n} \times \mathbb{R}: x_{n+1}=L(x) \fallingdotseq M_{0}+\xi \cdot\left(x-x_{0}\right)\right\}
$$

is a supporting hyperplane for $g$ at $x_{0}$ in $B_{6 \sqrt{n}}\left(x_{0}\right)$. Then $H$ has a parallel hyperplane $H^{\prime}$ which is a supporting hyperplane for $v$ in $B_{4 \sqrt{n}}$ at some point $x_{1} \in B_{2 \sqrt{n}}$. By the definition of concave envelope, we see that $H^{\prime}$ is also the hyperplane tangent to the graph of $\Gamma$ at $x_{1}$, so that $\xi=\nabla \Gamma\left(x_{1}\right)$. This implies that $B_{M_{0} / 6 \sqrt{n}}(0) \subset \nabla \Gamma\left(B_{2 \sqrt{n}}\right)$. Thus we have that

$$
C(n) \log \left(\frac{M_{0}^{n}}{\eta^{n}}\right) \leq \int_{C\left(u, \Gamma, B_{1}\right)} g_{\eta}(\nabla \Gamma(y)) \operatorname{det}\left[D^{2} \Gamma(y)\right]^{-} d y,
$$

where $g_{\eta}$ is the function given in Corollary 3.1.3. We also observe as shown in $[\mathrm{CC}]$ that

$$
\left|\nabla \Gamma\left(B_{2 \sqrt{n}} \backslash C\left(v, \Gamma, B_{2 \sqrt{n}}\right)\right)\right|=0 .
$$

Let $\left\{Q_{j}\right\}$ be the finite family of cubes given by Theorem 3.2 .1 (rescaled on $B_{2 \sqrt{n}}$ ). Then it follows from (4.2.1), (4.2.2) and Theorem 3.2.1 that

$$
\begin{aligned}
\ln \left(\frac{\left.\sup _{B_{2 \sqrt{n}}} v\right]^{n}}{\eta^{n}}+1\right) & \leq C \int_{C\left(u, \Gamma, B_{1}\right)} g_{\eta}(\nabla \Gamma(y)) \operatorname{det}\left[D^{2} \Gamma(y)\right]^{-} d y \\
& \leq C\left(\sum_{j} \sup _{\bar{Q}_{j}}\left(1+\eta^{-n}\left(\psi+\varepsilon_{0}\right)^{n}\right)\left|Q_{j}\right|\right) \\
& \leq C\left(\sum_{j}\left|Q_{j}\right|+\eta^{-n} \sum_{j} \sup _{\bar{Q}_{j}}\left(\psi+\varepsilon_{0}\right)^{n}\left|Q_{j}\right|\right) .
\end{aligned}
$$

If we set $\eta=\left(\sum_{j} \sup _{\bar{Q}_{j}}\left(\psi+\varepsilon_{0}\right)^{n}\left|Q_{j}\right|\right)^{1 / n}$ in (4.2.3), then we have that

$$
\sup _{B_{2 \sqrt{n}}} v \leq C\left(\sum_{j} \sup _{\bar{Q}_{j}}\left(\psi+\varepsilon_{0}\right)^{n}\left|Q_{j}\right|\right)^{1 / n} \leq C \varepsilon_{0}+C\left(\sum_{j}\left(\sup _{\bar{Q}_{j}} \psi\right)^{n}\left|Q_{j}\right|\right)^{1 / n} .
$$

Since $\inf _{Q_{1}} u \leq 1$ and $\inf _{Q_{1}} \Psi>2$, we see that $\sup _{B_{2 \sqrt{n}}} v>1$. If we choose $\eta$ and $\varepsilon_{0}$ small enough, the above inequality (4.2.4) implies that $1 / 2 \leq$ $C\left(\sum_{j}\left(\sup _{Q_{j}} \psi\right)^{n}\left|Q_{j}\right|\right)^{1 / n}$. We recall from the proof of Lemma 4.1.3 that $\psi$ is supported on $\bar{B}_{1 / 4}$ and bounded on $\mathbb{R}^{n}$. Thus the above inequality becomes $1 / 2 \leq C\left(\sum_{Q_{j} \cap B_{1 / 4} \neq \phi}\left|Q_{j}\right|\right)^{1 / n}$, which provides a lower bound for the sum of the volumes of the cubes $Q_{j}$ intersecting $B_{1 / 4}$ as follows;

$$
\sum_{Q_{j} \cap B_{1 / 4} \neq \phi}\left|Q_{j}\right| \geq c
$$


Since $\operatorname{diam}\left(Q_{j}\right) \leq \rho_{0} 2^{-\frac{1}{2-\sigma}} \leq \rho_{0}$ for any $\sigma \in\left(\sigma_{0}, 2\right)$, the cube $4 \sqrt{n} Q_{j}$ is contained in $B_{1 / 2}$ for any $Q_{j}$ with $Q_{j} \cap B_{1 / 4} \neq \phi$. Set $M_{1}=\sup _{B_{1 / 2}}(\Psi-\Gamma)$. Then by Theorem 3.2.1 we have that

$$
\left|\left\{y \in 4 \sqrt{n} Q_{j}: v(x) \geq \Gamma(y)-C d_{j}^{2}\right\}\right| \geq \gamma\left|Q_{j}\right|
$$

and $C d_{j}^{2} \leq C \rho_{0}^{2}$. Then the family $\mathfrak{F}=\left\{4 \sqrt{n} Q_{j}: Q_{j} \cap B_{1 / 4} \neq \phi\right\}$ is an open covering of the union $R \fallingdotseq \bigcup\left\{\bar{Q}_{j}: Q_{j} \cap B_{1 / 4} \neq \phi\right\}$. Now we may take a subcovering of $\mathfrak{F}$ with finite overlapping number (depending only on the dimension $n$ ) which covers the set $R$. Thus it follows from (4.2.5) and (4.2.6) that $\left|\left\{x \in B_{1 / 2}: v(x) \geq \Gamma(x)-C \rho_{0}^{2}\right\}\right| \geq v$. So we have that $\mid\left\{x \in B_{1 / 2}: u(x) \leq\right.$ $\left.M_{1}+C \rho_{0}^{2}\right\} \mid \geq v$. Taking $M=M_{1}+C \rho_{0}^{2}>1$, we conclude that $\left|\{u \leq M\} \cap Q_{1}\right| \geq v$ because $B_{1 / 2} \subset Q_{1}$. Hence we complete the proof.

Let $Q_{1}$ be the unit cube. Then we split it into $2^{n}$ cubes of half side. We do the same splitting step with each one of these $2^{n}$ cubes and we continue this process. The cubes obtained in this way are called dyadic cubes. If $Q$ is a dyadic cube different from $Q_{1}$, then we say that $\widetilde{Q}$ is the predecessor of $Q$ if $Q$ is one of $2^{n}$ cubes obtained from splitting $\widetilde{Q}$.

Lemma 4.2.2. $\left[\overline{C C}\right.$ Let $A$ and $B$ be measurable subsets of $\mathbb{R}^{n}$ with $A \subset B \subset Q_{1}$. If $\delta \in(0,1)$ is some number such that $(a)|A| \leq \delta$ and $(b) \widetilde{Q} \subset B$ for any dyadic cube $Q$ with $|A \cap Q|>\delta|Q|$, then $|A| \leq \delta|B|$.

4.3. Geometric Decay of Upper Level sets. The following lemma is a consequence of Lemma 2.4.2, Lemma 4.2.1 and Lemma 4.2.2

Lemma 4.3.1. Let $\varepsilon_{0}>0$ be the constant in Lemma 4.2.1. If $u \in \mathrm{B}\left(\mathbb{R}^{n}\right)$ is a viscosity supersolution to $\mathcal{M}_{\mathfrak{I}_{0}}^{-} u \leq \varepsilon_{0}$ on $B_{2 \sqrt{n}}$ such that $u \geq 0$ on $\mathbb{R}^{n}$ and $\inf _{Q_{1}} u \leq 1$, then there exist universal constants $C>0$ and $\varepsilon_{*}>0$ such that $\left|\{u>t\} \cap Q_{1}\right| \leq C t^{-\varepsilon_{*}}$ for any $t>0$.

Remark 4.3.2. We note that $B_{1 / 2} \subset Q_{1} \subset Q_{3} \subset B_{3 \sqrt{n} / 2} \subset B_{2 \sqrt{n}}$.

Proof. Note that $B_{1 / 2} \subset Q_{1} \subset Q_{3} \subset B_{3 \sqrt{n} / 2} \subset B_{2 \sqrt{n}}$. First, we shall prove that

$$
\left|\left\{u>M^{k}\right\} \cap Q_{1}\right| \leq(1-v)^{k}, \forall k \in \mathbb{N},
$$

where $v>0$ and $M>1$ are the constants chosen as in Lemma 4.2.1.

If $k=1$, then it has been done in Lemma 4.2.1. Assume the result (4.3.1) holds for $k-1(k \geq 2)$, and let $A=\left\{u>M^{k}\right\} \cap Q_{1}$ and $B=\left\{u>M^{k-1}\right\} \cap Q_{1}$. If we can show that $|A| \leq(1-v)|B|$, then (4.3.1) can be obtained for $k$. To show this, we apply Lemma 4.3.1. By Lemma 4.2.1, it is clear that $A \subset B \subset Q_{1}$ and $|A| \leq\left|\{u>M\} \cap Q_{1}\right| \leq 1-v$. So it remain only to prove (b) of Lemma 4.2.2. that is, we need to show that if $Q=Q_{2^{-i}}\left(x_{0}\right)$ is a dyadic cube satisfying

$$
|A \cap Q|>(1-v)|Q|
$$

then $\widetilde{Q} \subset B$. Indeed, we suppose that $\widetilde{Q} \not \subset B$ and take $x_{*} \in \widetilde{Q}$ such that

$$
u\left(x_{*}\right) \leq M^{k-1} .
$$


We now consider the transformation $x=x_{0}+2^{-i} y, y \in Q_{1}, x \in Q=Q_{2^{-i}}\left(x_{0}\right)$ and the function $v(y)=u(x) / M^{k-1}$. If we can show that $v$ satisfies the hypothesis of Lemma 4.2.1, then we have that $v<\left|\{v(y) \leq M\} \cap Q_{1}\right|=$ $2^{i n}\left|\left\{u(x) \leq M^{k}\right\} \cap Q\right|$, and thus $|Q \backslash A|>v|Q|$ which contradicts (4.3.2).

To complete the proof, we consider once again the transformation

$$
x=x_{0}+2^{-i} z, z \in B_{2 \sqrt{n}}, x \in B_{2 \sqrt{n} / 2^{i}}\left(x_{0}\right) \subset B_{2 \sqrt{n}}
$$

and the function $v(z)=u(x) / M^{k-1}$. It now remains to show that $v$ satisfies the hypothesis of Lemma 4.2.1. We now take any $\varphi \in C_{2 \sqrt{n}}^{2}(v ; z)^{-}$. If we set $\psi=M^{k-1} \varphi\left(2^{i}\left(\cdot-x_{0}\right)\right)$, then we observe that

$$
\varphi \in \mathrm{C}_{B_{2 \sqrt{n}}}^{2}(v ; z)^{-} \Leftrightarrow \psi \in \mathrm{C}_{B_{2 \sqrt{n} / 2^{i}\left(x_{0}\right)}^{2}\left(u ; x_{0}+2^{-i} z\right)^{-} .}
$$

If $K \in \mathcal{K}_{0}$, then we note that $K_{i} \in \mathcal{K}_{0}$ where $K_{i}(y)=2^{i(n+\sigma)} K\left(2^{i} y\right)$, and moreover the mapping $\mathcal{K}_{0} \rightarrow \mathcal{K}_{0}$ given by $K \mapsto K_{i}$ is an isometry. Since $B_{2 \sqrt{n} / 2^{i}}\left(x_{0}\right) \subset B_{2 \sqrt{n}}$, we have that

$$
\begin{aligned}
\mathcal{M}_{\mathfrak{I}_{0}}^{-} v(z ; \nabla \varphi) & \leq \mathcal{L}^{t} v(z ; \nabla \varphi)=\frac{1}{2^{i \sigma_{0}} M^{k-1}} \int_{\mathbb{R}^{n}} \mu_{t 2^{-i}}\left(u, x_{0}+2^{-i} z, y ; \nabla \psi\right) K_{i}(y) d y \\
& \fallingdotseq \frac{1}{2^{i \sigma_{0}} M^{k-1}} \mathcal{L}_{i}^{t 2^{-i}} u\left(x_{0}+2^{-i} z ; \nabla \psi\right)
\end{aligned}
$$

for any $\mathcal{L}^{t} \in \mathfrak{L}_{t}$ and any $t \geq 2^{i-1}$. Taking the infimum of the right-hand side in the above inequality, we obtain that

$$
\mathcal{M}_{\mathfrak{Q}_{0}}^{-} v(z ; \nabla \varphi) \leq \frac{1}{2^{i \sigma_{0}} M^{k-1}} \mathcal{M}_{\mathfrak{Q}_{0}}^{-} u\left(x_{0}+2^{-i} z ; \nabla \psi\right) .
$$

Thus $\mathcal{M}_{\mathfrak{I}_{0}}^{-} v(z ; \nabla \varphi) \leq \varepsilon_{0}$ because $\mathcal{M}_{\mathfrak{I}_{0}}^{-} u \leq \varepsilon_{0}$ on $B_{2 \sqrt{n}}$. By Theorem 2.2.4, we see that $\mathcal{M}_{\mathfrak{Q}_{0}}^{-} v \leq \varepsilon_{0}$ on $B_{2 \sqrt{n}}$. Also it is obvious that $v \geq 0$ on $\mathbb{R}^{n}$ and we see from (4.3.3) that $\inf _{Q_{1}} v \leq 1$. Finally the result follows immediately from (4.3.1) by taking $C=(1-v)^{-1}$ and $\varepsilon_{*}>0$ so that $1-v=M^{-\varepsilon_{*}}$. Hence we complete the proof.

By a standard covering argument we obtain the following theorem.

Theorem 4.3.3. For any $\sigma_{0} \in(1,2)$, let $\sigma \in\left(\sigma_{0}, 2\right)$ be given. If $u \in \mathrm{B}\left(\mathbb{R}^{n}\right)$ is a viscosity supersolution to $\mathcal{M}_{\mathfrak{I}_{0}}^{-} u \leq \varepsilon_{0}$ on $B_{2}$ such that $u \geq 0$ on $\mathbb{R}^{n}$ and $u(0) \leq 1$ where $\varepsilon_{0}$ is the constant given in Lemma 4.2.1, then there are universal constants $C>0$ and $\varepsilon_{*}>0$ such that $\left|\{u>t\} \cap B_{1}\right| \leq C t^{-\varepsilon_{*}}$ for any $t>0$.

In contrast to symmetric cases, we can not obtain the following theorem by rescaling the above theorem because our cases are not scaling invariant. We note that Theorem 4.3.4 on $r \in(0,1)$ shall be applied to obtain a Harnack inequality, and also Theorem 4.3.4 on $r \in[1,2]$ will be used to prove an interior $\mathrm{C}^{1, \alpha}$-regularity. 
Theorem 4.3.4. For any $\sigma_{0} \in(1,2)$, let $\sigma \in\left(\sigma_{0}, 2\right)$ be given, and let $x \in \mathbb{R}^{n}$ and $r \in(0,2]$. If $u \in \mathrm{B}\left(\mathbb{R}^{n}\right)$ is a viscosity supersolution to $\mathcal{M}_{\mathfrak{\Omega}_{0}}^{-} u \leq c_{0}$ on $B_{2 r}(x)$ such that $u \geq 0$ on $\mathbb{R}^{n}$, then there are universal constants $\varepsilon_{*}>0$ and $C>0$ such that

$$
\left|\{u>t\} \cap B_{r}(x)\right| \leq C r^{n}\left(u(x)+c_{0} r^{\sigma}\right)^{\varepsilon_{*}} t^{-\varepsilon_{*}} \text { for any } t>0 .
$$

Proof. Let $x \in \mathbb{R}^{n}$ and set $v(z)=u(r z+x) / q$ for $z \in B_{2}$ where $q=u(x)+c_{0} r^{\sigma} / \varepsilon_{0}$. Take any $\varphi \in C_{B_{2}}^{2}(v ; z)^{-}$. If we set $\psi=q \varphi((\cdot-x) / r)$, then we see that $\psi \in C_{B_{2 r}(x)}^{2}(u ; r z+x)$. Thus by the change of variables we have that

$$
\begin{aligned}
\mathcal{M}_{\mathfrak{I}_{0}}^{-} v(z ; \nabla \varphi) & \leq \mathcal{L}^{t} v(z ; \nabla \varphi)=\frac{r^{\sigma}}{q} \int_{\mathbb{R}^{n}} \mu_{t r}(r z+x, y ; \nabla \psi) K_{r}(y) d y \\
& \fallingdotseq \frac{r^{\sigma}}{q} \mathcal{L}_{r}^{t r} u(r z+x ; \nabla \psi)
\end{aligned}
$$

for any $\mathcal{L}^{t} \in \mathfrak{L}_{t}$ and any $t \geq 1 /(2 r)$, where $K_{r}(y)=r^{-n-\sigma} K(y / r)$ for $r \in(0,2]$. Taking the infimum of the right-hand side in the above inequality, we get

$$
\mathcal{M}_{\mathfrak{I}_{0}}^{-} v(z ; \nabla \varphi) \leq \frac{r^{\sigma}}{q} \mathcal{M}_{\mathfrak{I}_{0}}^{-} u(r z+x ; \nabla \psi) \leq \varepsilon_{0} .
$$

Thus by Theorem 3.4 we have that $\mathcal{M}_{\mathfrak{Q}_{0}}^{-} v \leq \varepsilon_{0}$ on $B_{2}$. Applying Theorem 4.3 .3 to the function $v$, we complete the proof.

\section{Regularities}

5.1. Harnack inequality. Harnack inequality plays an important role in analysis. In this section, we obtain the Harnack inequality for integrodifferential equations whose associated kernel is not necessarily symmetric. Our estimate depends only on a lower bound $\sigma_{0} \in(1,2)$ for $\sigma \in\left(\sigma_{0}, 2\right)$ and also remains uniform as $\sigma \rightarrow 2$. In this respect, we can look upon this estimate as a generalization of Krylov-Safonov Harnack inequality. The proof is an adaptation of the method used in [CS] to the case whose associated kernel is not necessarily symmetric.

Theorem 5.1.1. For $\sigma_{0} \in(1,2)$, let $\sigma_{0}<\sigma<2$. If $u \in \mathrm{B}\left(\mathbb{R}^{n}\right)$ is a positive function such that

$$
\mathcal{M}_{\mathfrak{Q}_{0}}^{-} u \leq C_{0} \text { and } \mathcal{M}_{\mathfrak{I}_{0}}^{+} u \geq-C_{0} \text { on } B_{2}
$$

in the viscosity sense, then there is some constant $C>0$ depending only on $\lambda, \Lambda, n$ and $\sigma_{0}$ such that

$$
\sup _{B_{1 / 2}} u \leq C\left(\inf _{B_{1 / 2}} u+C_{0}\right)
$$

Proof. Let $\hat{x} \in B_{1 / 2}$ be a point so that $\inf _{B_{1 / 2}} u=u(\hat{x})$. Then it is enough to show that $\sup _{B_{1 / 2}} u \leq C\left(u(\hat{x})+C_{0}\right)$. Without loss of generality, we may assume that $u(\hat{x}) \leq 1$ and $C_{0}=1$ by dividing $u$ by $u(\hat{x})+C_{0}$. Let $\varepsilon_{*}>0$ be the number given in Theorem 4.3.4 and let $\beta=n / \varepsilon_{*}$. We now set $s_{0}=\inf \{s>0$ : $\left.u(x) \leq s(1-|x|)^{-\beta}, \forall x \in B_{1}\right\}$. Then we see that $s_{0}>0$ because $u$ is positive on 
$\mathbb{R}^{n}$. Also there is some $\check{x} \in B_{1}$ such that $u(\check{x})=s_{0}(1-|\check{x}|)^{-\beta}=s_{0} \mathrm{~d}_{0}^{-\beta}$ where $\mathrm{d}_{0}=\mathrm{d}\left(\check{x}, \partial B_{1}\right) \leq 1$.

To finish the proof, we have only to show that $s_{0}$ can not be too large because $u(x) \leq C_{1}(1-|x|)^{-\beta} \leq C$ for any $x \in B_{1 / 2}$ if $C_{1}>0$ is some constant with $s_{0} \leq C_{1}$. Assume that $s_{0}$ is very large. Then by Theorem 4.3 .3 we have that

$$
\left.\mid\{u \geq u(\check{x}) / 2\} \cap B_{1}\right\}\left.|\leq| \frac{2}{u(\check{x})}\right|^{\varepsilon_{*}} \leq C s_{0}^{-\varepsilon_{*}} \mathrm{~d}_{0}^{n} .
$$

Since $\left|B_{r}\right|=C \mathrm{~d}_{0}^{n}$ for $r=\mathrm{d}_{0} / 2<1$, we easily obtain that

$$
\left.\mid\{u \geq u(\check{x}) / 2\} \cap B_{r}(\check{x})\right\}\left.|\leq| \frac{2}{u(\check{x})}\right|^{\varepsilon_{*}} \leq C s_{0}^{-\varepsilon_{*}}\left|B_{r}\right| .
$$

In order to get a contradiction, we estimate $\left|\{u \leq u(\check{x}) / 2\} \cap B_{\delta r}(\check{x})\right|$ for some very small $\delta>0$ (to be determined later). For any $x \in B_{2 \delta r}(\check{x})$, we have that $u(x) \leq s_{0}\left(\mathrm{~d}_{0}-\delta \mathrm{d}_{0}\right)^{-\beta} \leq u(\check{x})(1-\delta)^{-\beta}$ for $\delta>0$ so that $(1-\delta)^{-\beta}$ is close to 1.

We consider the function $v(x)=(1-\delta)^{-\beta} u(\check{x})-u(x)$. Then we see that $v \geq 0$ on $B_{2 \delta r}(\check{x})$, and also $\mathcal{M}_{\mathfrak{Q}_{0}}^{-} v \leq 1$ on $B_{\delta r}(\check{x})$ because $\mathcal{M}_{\mathfrak{I}_{0}}^{+} u \geq-1$ on $B_{\delta r}(\check{x})$. We now want to apply Theorem 4.3.4 to $v$. However $v$ is not positive on $\mathbb{R}^{n}$ but only on $B_{\delta r}(\check{x})$. To apply Theorem 4.3.4, we consider $w=v^{+}$ instead of $v$. Since $w=v+v^{-}$, we have that $\mathcal{M}_{\mathfrak{I}_{0}}^{-} w \leq \mathcal{M}_{\mathfrak{I}_{0}}^{-} v+\mathcal{M}_{\mathfrak{I}_{0}}^{+} v^{-} \leq$ $1+\mathcal{M}_{\mathfrak{I}_{0}}^{+} v^{-}$on $B_{\delta r}(\check{x})$. Since $v^{-} \equiv 0$ on $B_{2 \delta r}(\check{x})$, if $x \in B_{\delta r}(\check{x})$ then we have that $\mu_{t}\left(v^{-}, x, y ; \nabla \varphi\right)=v^{-}(x+y)$ for any $t \geq 1 / 2, y \in B_{\delta r}(\check{x})$ and $\varphi \in C_{B_{\delta r}(\check{x})}^{2}\left(v^{-} ; x\right)^{+}$. Take any $\varphi \in C_{B_{\delta r}(\check{x})}^{2}\left(v^{-} ; x\right)^{+}$and any $x \in B_{\delta r}(\check{x})$. Since $x+B_{\delta r} \subset B_{2 \delta r}(\check{x})$, we thus have that

$$
\begin{aligned}
\mathcal{M}_{\mathfrak{I}_{0}}^{-} & w(x ; \nabla \varphi) \\
& \leq 1+(2-\sigma) \int_{\mathbb{R}^{n}} \frac{\Lambda \mu_{t}^{+}\left(v^{-}, x, y ; \nabla \varphi\right)-\lambda \mu_{t}^{-}\left(v^{-}, x, y ; \nabla \varphi\right)}{|y|^{n+\sigma}} d y \\
& \leq 1+(2-\sigma) \int_{\left\{y \in \mathbb{R}^{n}: v(x+y)<0\right\}} \frac{-\Lambda v(x+y)}{|y|^{n+\sigma}} d y \\
& \leq 1+(2-\sigma) \Lambda \int_{\mathbb{R}^{n} \backslash B_{\delta r}} \frac{\left(u(x+y)-(1-\delta)^{-\beta} u(\check{x})\right)_{+}}{|y|^{n+\sigma}} d y .
\end{aligned}
$$

Set $h_{c}(x)=c\left(1-|x|^{2}\right)_{+}$for $c>0$ and $c_{1}=\sup \left\{c>0: u(x) \geq h_{c}(x), \forall x \in \mathbb{R}^{n}\right\}$. Then there is some $x_{1} \in B_{1}$ such that $u\left(x_{1}\right)=c_{1}\left(1-\left|x_{1}\right|^{2}\right)$ and we see that $c_{1} \leq 4 / 3$ because $u(\hat{x}) \leq 1$. Since $\nabla h_{c_{1}}(x)=-2 c_{1} x$, we have that

$$
\begin{aligned}
(2-\sigma) \int_{\mathbb{R}^{n}} \frac{\mu_{t}^{-}\left(u, x_{1}, y ; \nabla h_{c_{1}}\right)}{|y|^{n+\sigma}} d y & \leq(2-\sigma) \int_{\mathbb{R}^{n}} \frac{\mu_{t}^{-}\left(h_{\mathcal{c}_{1}}, x_{1}, y\right)}{|y|^{n+\sigma}} d y \\
& \leq \frac{8\left(2-\sigma_{0}\right)}{3} \int_{\mathbb{R}^{n}} \frac{1+|y|}{|y|^{n+\sigma_{0}}} d y \leq C
\end{aligned}
$$


for some constant $C>0$ which is independent of $\sigma$, and so we have that

$$
\Lambda(2-\sigma) \sup _{t \geq 1 / 2} \int_{\mathbb{R}^{n}} \frac{\mu_{t}^{-}\left(u, x_{1}, y ; \nabla h_{c_{1}}\right)}{|y|^{n+\sigma}} d y \leq C .
$$

Since $\mathcal{M}_{\mathfrak{Q}_{0}}^{-} u\left(x_{1}\right) \leq 1$ on $B_{2}$, by (5.1.3) we have that

$$
\begin{aligned}
1 \geq \mathcal{M}_{\mathfrak{I}_{0}}^{-} u\left(x_{1} ; \nabla h_{c_{1}}\right) & \geq \lambda(2-\sigma) \inf _{t \geq 1 / 2} \int_{\mathbb{R}^{n}} \frac{\mu_{t}^{+}\left(u, x_{1}, y ; \nabla h_{c_{1}}\right)}{|y|^{n+\sigma}} d y \\
& -\Lambda(2-\sigma) \sup _{t \geq 1 / 2} \int_{\mathbb{R}^{n}} \frac{\mu_{t}^{-}\left(u, x_{1}, y ; \nabla h_{c_{1}}\right)}{|y|^{n+\sigma}} d y .
\end{aligned}
$$

Thus we obtain that $(2-\sigma) \inf _{t \geq 1 / 2} \int_{\mathbb{R}^{n}} \frac{\mu_{t}^{+}\left(u, x_{1}, y ; \nabla h_{c_{1}}\right)}{|y|^{n+\sigma}} d y \leq C$ for a constant $C>0$ which is independent of $\sigma$, and so there is some $t_{1} \geq 1 / 2$ such that

$$
(2-\sigma) \int_{\mathbb{R}^{n}} \frac{\mu_{t_{1}}^{+}\left(u, x_{1}, y ; \nabla h_{c_{1}}\right)}{|y|^{n+\sigma}} d y \leq C .
$$

Since $\mu_{t}\left(u, x_{1}, y ; \nabla h_{c_{1}}\right) \geq u\left(x_{1}+y\right)-4 / 3-8 t / 3$ for any $t \geq 1 / 2$ and $y \in B_{t}$, and

$$
\left(u\left(x_{1}+y\right)-4 / 3-8 t / 3\right)_{+}=0 \text { for any } t \geq \frac{3}{8} \sup _{y \in \mathbb{R}^{n}}\left[u\left(x_{1}+y\right)-4 / 3\right],
$$

we may assume that $t_{1} \geq 1 / 2$ must be finite. Since $\mu_{t_{1}}^{+}\left(u, x_{1}, y ; \nabla h_{c_{1}}\right) \geq$ $\left(u\left(x_{1}+y\right)-4 / 3-8 t_{1} / 3\right)_{+}$for any $y \in \mathbb{R}^{n}$, by (5.1.4) we obtain that

$$
\begin{aligned}
(2-\sigma) \int_{\mathbb{R}^{n}} & \frac{\left(u\left(x_{1}+y\right)-4 / 3-8 t_{1} / 3\right)_{+}}{|y|^{n+\sigma}} d y \\
& \leq(2-\sigma) \int_{\mathbb{R}^{n}} \frac{\mu_{t_{1}}^{+}\left(u, x_{1}, y ; \nabla h_{c_{1}}\right)}{|y|^{n+\sigma}} d y \leq C .
\end{aligned}
$$

We now may assume that $(1-\delta)^{-\beta} u(\check{x})=(1-\delta)^{-\beta} S_{0}(1-|\check{x}|)^{-\beta} \geq 4 / 3+8 t_{1} / 3$ because $s_{0}$ was very large and $(1-\delta)^{-\beta}$ was close to 1 . Since $\delta r<1$, by (5.1.5) and the change of variables we have that

$$
\begin{aligned}
& (2-\sigma) \Lambda \int_{B_{\delta r}^{c}} \frac{\left(u(x+y)-(1-\delta)^{-\beta} u(\check{x})\right)_{+}}{|y|^{n+\sigma}} d y \\
& \leq(2-\sigma) \Lambda \int_{B_{\delta r}^{c} \cap B_{100}} \frac{\left(u\left(x_{1}+y+x-x_{1}\right)-(1-\delta)^{-\beta} u(\check{x})\right)_{+}}{\left|y+x-x_{1}\right|^{n+\sigma}} \frac{\left|y+x-x_{1}\right|^{n+\sigma}}{|y|^{n+\sigma}} d y \\
& +(2-\sigma) \Lambda \int_{B_{\delta r}^{c} \cap B_{100}^{c}} \frac{\left(u\left(x_{1}+y+x-x_{1}\right)-(1-\delta)^{-\beta} u(\check{x})\right)_{+}}{\left|y+x-x_{1}\right|^{n+\sigma}} \frac{\left|y-x_{1}\right|^{n+\sigma}}{|y|^{n+\sigma}} d y \\
& \leq C\left((\delta r)^{-n-\sigma}+1\right)(2-\sigma) \Lambda \int_{\mathbb{R}^{n}} \frac{\left(u\left(x_{1}+y\right)-4 / 3-8 t_{1} / 3\right)_{+}}{|y|^{n+\sigma}} d x \leq C(\delta r)^{-n-\sigma}
\end{aligned}
$$


for any $x \in B_{\delta r}(\check{x})$. Thus by (5.1.2) and Theorem 2.2.4 we obtain that

$$
\mathcal{M}_{\mathfrak{Q}_{0}}^{-} w(x) \leq C(\delta r)^{-n-\sigma} \text { on } B_{\delta r}(\check{x}) \text {. }
$$

Since $u(\check{x})=s_{0} \mathrm{~d}_{0}^{-\beta}=2^{-\beta} s_{0} r^{-\beta}$ and $\beta \varepsilon_{*}=n$, applying Theorem 4.3.4 we have

$$
\begin{aligned}
\mid\{u \leq & u(\check{x}) / 2\} \cap B_{\delta r / 2}(\check{x})|=|\left\{w \geq u(\check{x})\left((1-\delta)^{-\beta}-1 / 2\right)\right\} \cap B_{\delta r / 2}(\check{x}) \mid \\
& \leq C(\delta r)^{n}\left[\left((1-\delta)^{-\beta}-1\right) u(\check{x})+C(\delta r)^{-n-\sigma}(\delta r)^{\sigma}\right]^{\varepsilon_{*}}\left[u(\check{x})\left((1-\delta)^{-\beta}-1 / 2\right)\right]^{-\varepsilon_{*}} \\
& \leq C(\delta r)^{n}\left[\left((1-\delta)^{-\beta}-1\right)^{\varepsilon_{*}}+\delta^{-n \varepsilon_{*}} S_{0}^{-\varepsilon_{*}}\right] .
\end{aligned}
$$

We now choose $\delta>0$ so small enough that $C(\delta r)^{n}\left((1-\delta)^{-\beta}-1\right)^{\varepsilon_{*}} \leq\left|B_{\delta r / 2}(\check{x})\right| / 4$. Since $\delta$ was chosen independently of $s_{0}$, if $s_{0}$ is large enough for such fixed $\delta$ then we get that $C(\delta r)^{n} \delta^{-n \varepsilon_{*}} s_{0}^{-\varepsilon_{*}} \leq\left|B_{\delta r / 2}(\check{x})\right| / 4$. Therefore we obtain that $\left|\{u \leq u(\check{x}) / 2\} \cap B_{\delta r / 2}(\check{x})\right| \leq\left|B_{\delta r / 2}(\check{x})\right| / 2$. Thus we conclude that

$$
\begin{aligned}
\left|\{u \geq u(\check{x}) / 2\} \cap B_{r}(\check{x})\right| & \geq\left|\{u \geq u(\check{x}) / 2\} \cap B_{\delta r / 2}(\check{x})\right| \geq\left|\{u>u(\check{x}) / 2\} \cap B_{\delta r / 2}(\check{x})\right| \\
& \geq\left|B_{\delta r / 2}(\check{x})\right|-\left|B_{\delta r / 2}(\check{x})\right| / 2=\left|B_{\delta r / 2}(\check{x})\right| / 2=C\left|B_{r}\right|,
\end{aligned}
$$

which contradicts (5.1.1) if $s_{0}$ is large enough. Thus we complete the proof.

5.2. Hölder estimates. The purpose of this section is to prove the following Hölder regularity result (see Theorem 5.2.2). Before doing this, we obtain a technical lemma which shall be useful in proving Theorem 5.2.2.

Lemma 5.2.1. For any $\sigma_{0} \in(1,2)$, let $\sigma \in\left(\sigma_{0}, 2\right)$ be given. If $u$ is a bounded function with $|u| \leq 1 / 2$ on $\mathbb{R}^{n}$ such that

$$
\mathcal{M}_{\mathfrak{S}_{0}}^{+} u \geq-\varepsilon_{0} \text { and } \mathcal{M}_{\mathfrak{I}_{0}}^{-} u \leq \varepsilon_{0} \text { on } B_{1}
$$

in the viscosity sense where $\varepsilon_{0}>0$ is some sufficiently small constant, then there is some universal constant $\alpha>0$ (depending only on $\lambda, \Lambda, n$ and $\sigma_{0}$ ) such that $u \in C^{\alpha}$ at the origin. More precisely, $|u(x)-u(0)| \leq C|x|^{\alpha}$ for some universal constant $C>0$ depending only on $\alpha$.

We shall prove the following theorem using only Theorem 4.3.4. Theorem 5.2.2 easily follows from Lemma 5.2.1 by a simple rescaling argument. Thus we have only to prove Lemma 5.2.1 to establish Theorem 5.2.2.

Theorem 5.2.2. For any $\sigma_{0} \in(1,2)$, let $\sigma \in\left(\sigma_{0}, 2\right)$ be given. If $u$ is a bounded function on $\mathbb{R}^{n}$ such that $\mathcal{M}_{\mathfrak{L}_{0}}^{+} u \geq-c_{0}$ and $\mathcal{M}_{\mathfrak{I}_{0}}^{-} u \leq c_{0}$ on $B_{1}$ in the viscosity sense, then there is a constant $\alpha>0$ (depending only on $\lambda, \Lambda, n$ and $\sigma_{0}$ ) such that

$$
\|u\|_{C^{\alpha}\left(B_{1 / 2}\right)} \leq C\left(\|u\|_{L^{\infty}\left(\mathbb{R}^{n}\right)}+c_{0}\right)
$$

where $C>0$ is some universal constant depending only on $\alpha$.

[Proof of Lemma 5.2.1] Take any $\alpha \in\left(0, \sigma_{0}\right)$ and choose an $N$ so large that

$$
\frac{2^{\sigma_{0}+1}\left(2-\sigma_{0}\right) \Lambda \omega_{n}}{\sigma_{0}-\alpha} 2^{-\left(\sigma_{0}-\alpha\right) N} \leq \varepsilon_{0} / 2 \text { and } 2^{1-\sigma_{0} N} 2^{-k\left(\sigma_{0}-\alpha\right) N} \leq 1 / 2
$$


Then it suffices to show that there exist a nondecreasing sequence $\left\{m_{k}\right\}_{k \in \mathbb{N} \cup\{0\}}$ and a nonincreasing sequence $\left\{M_{k}\right\}_{k \in \mathbb{N} \cup\{0\}}$ such that $m_{k} \leq u \leq M_{k}$ in $B_{2^{-k N}}$ and $M_{k}-m_{k}=2^{-\alpha k N}$, so that the theorem holds with $C=2^{\alpha N}$; for, if $2^{-(k+1) N} \leq|x| \leq 2^{-k N}$ for $k \in \mathbb{N} \cup\{0\}$, then

$$
|u(x)-u(0)| \leq \frac{M_{k}-m_{k}}{2^{-\alpha(k+1) N}} \cdot 2^{-\alpha(k+1) N} \leq 2^{\alpha N}|x|^{\alpha} .
$$

We construct $m_{k}$ and $M_{k}$ by induction. For $k \leq 0$, we can take $m_{k}=\inf _{\mathbb{R}^{n}} u$ and $M_{k}=m_{k}+2^{-\alpha k N}$ because $\operatorname{osc}_{\mathbb{R}^{n}} u \leq 1$. Assume that we have the sequences up to $m_{k}$ and $M_{k}$ for $k \geq 1$. Then we want to show that we can continue the sequences by finding $m_{k+1}$ and $M_{k+1}$. Fix any $z \in B_{1 / \tau}$ where $\tau=2^{-(k+1) N}$. Take any $\varphi \in C_{B_{1 / \tau}}^{2}(v ; z)^{-}$where $v=\frac{u(\tau \cdot)-m_{k}}{\left(M_{k}-m_{k}\right) / 2}$. If we set $\psi=m_{k}+\frac{M_{k}-m_{k}}{2} \varphi(\cdot / \tau)$, then we see that $\psi \in \mathrm{C}_{B_{1}}^{2}(u ; \tau z)^{-}$and moreover we have that

$$
\nabla \psi(\tau z)=\frac{M_{k}-m_{k}}{2 \tau} \nabla \varphi(z)
$$

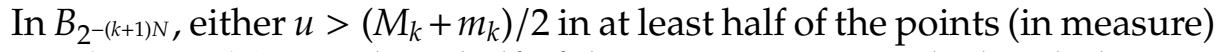
or $u \leq\left(M_{k}+m_{k}\right) / 2$ in at least half of the points. First, we deal with the case

$$
\left|\left\{u>\left(M_{k}+m_{k}\right) / 2\right\} \cap B_{2^{-(k+1) N}}\right| \geq\left|B_{2^{-(k+1) N}}\right| / 2 .
$$

Then we note that $v \geq 0$ on $B_{2^{N}}$ and $\left|\{v>1\} \cap B_{1}\right| \geq\left|B_{1}\right| / 2$. Then we have

$$
\begin{aligned}
\mathcal{M}_{\mathfrak{I}_{0}}^{-} v(z ; \nabla \varphi) & \leq \mathcal{L}^{t} v(z ; \nabla \varphi)=\frac{2 \tau^{\sigma}}{M_{k}-m_{k}} \int_{\mathbb{R}^{n}} \mu_{\tau \tau}(u, \tau z, y ; \nabla \psi) K_{\tau}(y) d y \\
& \fallingdotseq \frac{2 \tau^{\sigma}}{M_{k}-m_{k}} \mathcal{L}_{\tau}^{t \tau} u(\tau z ; \nabla \psi)
\end{aligned}
$$

for any $\mathcal{L}^{t} \in \mathfrak{L}_{t}$ and any $t \geq 2^{(k+1) N-1}=1 /(2 \tau)$. Taking the infimum of the right-hand side in the above inequality, we obtain that

$$
\mathcal{M}_{\mathfrak{I}_{0}}^{-} v(z ; \nabla \varphi) \leq \frac{2 \tau^{\sigma}}{M_{k}-m_{k}} \mathcal{M}_{\mathfrak{I}_{0}}^{-} u(\tau z ; \nabla \psi)
$$

Since $\mathcal{M}_{\mathfrak{Q}_{0}}^{-} u \leq \varepsilon_{0}$ on $B_{1}$ in the viscosity sense, we have that

$$
\mathcal{M}_{\mathfrak{Q}_{0}}^{-} v(z ; \nabla \varphi) \leq \frac{2 \tau^{\sigma}}{M_{k}-m_{k}} \mathcal{M}_{\mathfrak{I}_{0}}^{-} u(\tau z ; \nabla \psi) \leq 2^{1-\sigma_{0} N} 2^{-k\left(\sigma_{0}-\alpha\right) N} \varepsilon_{0} \leq \varepsilon_{0} / 2 .
$$

Thus this implies that

$$
\mathcal{M}_{\mathfrak{I}_{0}}^{-} v \leq \varepsilon_{0} / 2 \text { on } B_{2^{(k+1) N}} .
$$

It also follows from the inductive hypothesis that if $2^{j N} \leq|x| \leq 2^{(j+1) N}$ then

$$
\begin{aligned}
& v(x) \geq \frac{m_{k-j}-M_{k-j}+M_{k}-m_{k}}{\left(M_{k}-m_{k}\right) / 2} \geq 2\left(1-|x|^{\alpha}\right), \\
& v(x) \leq \frac{M_{k-j}-m_{k-j}+m_{k-j}-m_{k}}{\left(M_{k}-m_{k}\right) / 2} \leq 2|x|^{\alpha} .
\end{aligned}
$$


for any $j \in \mathbb{N} \cup\{0\}$. That is to say, $-2\left(|x|^{\alpha}-1\right) \leq v(x) \leq 2|x|^{\alpha}$ outside $B_{1}$.

If we set $w(x)=\max \{v(x), 0\}$, then we have that $\mathcal{M}_{\mathfrak{L}_{0}}^{-} w \leq 4 \varepsilon_{0}$ on $B_{2^{N-1}}$; for, if $x \in B_{2^{N-1}}$ and $y \in B_{2^{N-1}}$, then $\mu_{t}\left(v^{-}, x, y\right)=0$ for any $t \geq 1 / 2$ because $v \geq 0$ on $B_{2^{N}}$; if $x \in B_{2^{N-1}}$ and $y \in B_{2^{N-1}}^{c}$, then $\mu_{t}\left(v^{-}, x, y\right)=v^{-}(x+y)$ for any $t \geq 1 / 2$ because $v \geq 0$ on $B_{2^{N}}$. Since $w=v+v^{-}$, we see that $\mathcal{M}_{\mathfrak{Q}_{0}}^{-} w \leq \mathcal{M}_{\mathfrak{Q}_{0}}^{-} v+\mathcal{M}_{\mathfrak{Q}_{0}}^{+} v^{-}$. Thus it follows from (5.2.1) and (5.2.4) that if $x \in B_{2^{N-1}}$ is given, then

$$
\begin{aligned}
\mathcal{L}^{t} v^{-}(x) & \leq \int_{\mathbb{R}^{n}} \mu_{t}\left(v^{-}, x, y\right) K(y) d y \leq(2-\sigma) \Lambda \int_{|y| \geq 2^{N-1}} \frac{2|x+y|^{\alpha}}{|y|^{n+\sigma}} d y \\
& \leq\left(2-\sigma_{0}\right) \Lambda \int_{|y| \geq 2^{N-1}} \frac{2^{\alpha+1}}{|y|^{n+\sigma_{0}-\alpha}} d y=\frac{2^{\sigma_{0}+1}\left(2-\sigma_{0}\right) \Lambda \omega_{n}}{\sigma_{0}-\alpha} 2^{-\left(\sigma_{0}-\alpha\right) N} \leq \varepsilon_{0} / 2
\end{aligned}
$$

for any $\mathcal{L}^{t} \in \mathfrak{L}_{0}$, whenever $\alpha \in\left(0, \sigma_{0}\right)$ and $\sigma \in\left(\sigma_{0}, 2\right)$. So we have that $\mathcal{M}_{\mathfrak{I}_{0}}^{+} v^{-} \leq \varepsilon_{0} / 2$ on $B_{2^{N-1}}$. Thus by (5.2.3) we conclude that $\mathcal{M}_{\mathfrak{I}_{0}}^{-} w \leq \varepsilon_{0}$ on $B_{2^{N-1}}$ where $\alpha \in\left(0, \sigma_{0}\right)$.

Take any point $x \in B_{1}$. Since $B_{1} \subset B_{2}(x) \subset B_{4}(x) \subset B_{2^{N-1}}$, we can apply Theorem 4.3.4 on $B_{2}(x)$ to obtain that $2^{n} C\left(w(x)+2^{\sigma} \varepsilon_{0}\right)^{\varepsilon_{*}} \geq\left|\{w>1\} \cap B_{2}(x)\right| \geq$ $\left|\{v>1\} \cap B_{1}\right| \geq \frac{1}{2}\left|B_{1}\right|$. Thus we have that $w(x) \geq\left(\frac{\left|B_{1}\right|}{2^{n+1} C}\right)^{1 / \varepsilon_{*}}-4 \varepsilon_{0}$ for any $x \in B_{1}$. If we choose $\varepsilon_{0}$ sufficiently small, then this implies that $w \geq \vartheta$ on $B_{1}$ for some $\vartheta>0$. If we set $M_{k+1}=M_{k}$ and $m_{k+1}=m_{k}+\vartheta\left(M_{k}-m_{k}\right) / 2$, then we have that $m_{k+1} \leq u \leq M_{k+1}$ in $B_{2-(k+1) N}$. Moreover, $M_{k+1}-m_{k+1}=$ $(1-\vartheta / 2) 2^{-\alpha k N}$. Then we may choose some small $\alpha>0$ and $\vartheta>0$ so that $1-\vartheta / 2=2^{-\alpha N}$, so that we obtain that $M_{k+1}-m_{k+1}=2^{-\alpha(k+1) N}$.

On the other hand, if we deal with the second case

$$
\left|\left\{u \leq\left(M_{k}+m_{k}\right) / 2\right\} \cap B_{2^{-(k+1) N}}\right| \geq\left|B_{2^{-(k+1) N}}\right| / 2,
$$

then we consider the function $v(x)=\frac{M_{k}-u\left(2^{-(k+1) N} x\right)}{\left(M_{k}-m_{k}\right) / 2}$ and repeat in the same way by using $\mathcal{M}_{\mathfrak{I}_{0}}^{+} u \geq-\varepsilon_{0}$.

5.3. $C^{1, \alpha}$-estimates. In this section, we prove an interior $C^{1, \alpha}$-regularity result for viscosity solutions to a general class of fully nonlinear integrodifferential equations. The key idea of proof is to apply the Hölder estimates of Theorem 5.2.2 to incremental quotients of the solution. There being no uniform bound in $L^{\infty}$ for the incremental quotients outside the domain may cause a technical difficulty since we are dealing with nonlocal equations. We shall solve it by assuming some extra regularity of the family of linear integro-differential operators $\mathcal{L}^{t}$. The extra assumption, added to the growth condition (2.1.3) for the kernel, is a modulus of continuity of $K$ in measure so that far away oscillations tend to cancel out. 
We consider the class $\mathfrak{L}_{0}^{1}$ consisting of the operators $\mathcal{L}^{t} \in \mathfrak{L}_{0}$ associated with kernels $K$ for which (2.1.3) holds and there exists some $\varrho_{1}>0$ such that

$$
\sup _{h \in B_{\varrho_{1} / 2}} \int_{\mathbb{R}^{n} \backslash B_{\varrho_{1}}} \frac{|K(y)-K(y-h)|}{|h|} d y \leq C .
$$

If $K$ is a radial function satisfying (2.1.3), then it is interesting that the condition (5.3.1) is not required. Indeed, if $K(y)=(2-\sigma) A /|y|^{n+\sigma}$ for $\lambda \leq$ $A \leq \Lambda$, then it follows from the mean value theorem and Schwartz inequality that

$$
\sup _{h \in B_{\varrho_{1} / 2}} \int_{\mathbb{R}^{n} \backslash B_{\varrho_{1}}} \frac{|K(y)-K(y-h)|}{|h|} d y=(2-\sigma) \Lambda(n+\sigma) 2^{n+\sigma+1} \frac{\omega_{n}}{\sigma+1} \varrho_{1}^{-1-\sigma} \leq C .
$$

for any $h \in B_{\varrho_{1} / 2}$ and $y \in \mathbb{R}^{n} \backslash B_{\varrho_{1}}$, because $|y| \geq 2|h|$ for such $h, y$ and $|y-\tau h| \geq|y|-|h| \geq|y|-|y| / 2=|y| / 2$ for $\tau \in[0,1]$.

In the following theorem, we shall furnish interior $C^{1, \alpha}$-estimates for fully nonlinear elliptic equations associated with a class of linear integrodifferential operators.

Theorem 5.3.1. For any $\sigma_{0} \in(1,2)$, let $\sigma \in\left(\sigma_{0}, 2\right)$ be given. Then there is some $\varrho_{1}>0$ (depending on $\lambda, \Lambda, \sigma_{0}$ and the dimension $n$ ) so that if $\mathcal{I}^{ \pm}$is a nonlocal elliptic operator with respect to $\mathfrak{Q}_{0}^{1}$ in the sense of Definition 2.2.1 and $u \in \mathrm{B}\left(\mathbb{R}^{n}\right)$ is a viscosity solution to $\mathcal{I}^{ \pm} u=0$ on $B_{1}$, then there is a universal constant $\alpha>0$ (depending only on $\lambda, \Lambda, \sigma_{0}$ and the dimension $n$ ) such that

$$
\|u\|_{C^{1, \alpha}\left(B_{1 / 2}\right)} \leq C\left(\|u\|_{L^{\infty}\left(\mathbb{R}^{n}\right)}+\left|\mathcal{I}^{ \pm} 0\right|\right)
$$

for some constant $C>0$ depending on $\lambda, \Lambda, \sigma_{0}, n$ and the constant given in (10.1) (where we denote by $\mathcal{I}^{ \pm} 0$ the value we obtain when we apply $\mathcal{I}^{ \pm}$to the constant function that is equal to zero).

Proof. Since $\mathcal{I}^{ \pm} u=0$ on $B_{1}$, it follows from Lemma 2.2.2 that $\mathcal{M}_{\mathfrak{Q}_{0}}^{+} u \geq$ $\mathcal{I}^{ \pm} u-\mathcal{I}^{ \pm} 0=-\mathcal{I}^{ \pm} 0 \geq-\left|\mathcal{I}^{ \pm} 0\right|$ on $B_{1}$. Similarly we have that $\mathcal{M}_{\mathfrak{L}_{0}}^{-} u \leq\left|\mathcal{I}^{ \pm} 0\right|$ on $B_{1}$. Thus by Theorem 5.2.2 we see that $u \in C^{\alpha}\left(B_{1-\delta}\right)$ for any $\delta \in(0,1)$ and $\|u\|_{C^{\alpha}\left(B_{1-\delta}\right)} \leq C\left(\|u\|_{L^{\infty}\left(\mathbb{R}^{n}\right)}+\left|\mathcal{I}^{ \pm} 0\right|\right)$. Now we want to improve the obtained regularity iteratively by applying Theorem 5.2.2 again until we obtain Lipschitz regularity in a finite number of steps.

Assume that we have proven that $u \in C^{\beta}\left(B_{r}\right)$ for some $\beta \in(0,1]$ and $r \in(0,1)$. Then we apply Theorem 5.2.2 for the difference quotient $w^{h}=$ $\left(\tau_{h} u-u\right) /|h|^{\beta}$ where $\tau_{h}$ is a translation operator given by $\tau_{h} u(x)=u(x+h)$ for $h \in \mathbb{R}^{n}$. Since $\mathcal{M}_{\mathfrak{I}_{0}}^{+} u=-\mathcal{M}_{\mathfrak{I}_{0}}^{-}[-u]$, we see from Theorem 2.4.4 that $\mathcal{M}_{\mathfrak{Q}_{0}}^{+} w^{h} \geq 0$ and $\mathcal{M}_{\mathfrak{Q}_{0}}^{-} w^{h} \leq 0$ on $B_{r}$ for any $h \in(0,1-r)$. Since $u \in C^{\beta}\left(B_{r}\right)$, we see that the family $\left\{w^{h}\right\}_{|h| \in(0,1-r)}$ is uniformly bounded on $B_{r}$. But the functions $w^{h}$ is not uniformly bounded outside the ball $B_{r}$, and so we can not apply Theorem 5.2.2 directly. However we observe from (5.3.1) that $w^{h}$ has oscillations that give cancelations in the integrals. Let $\phi$ be a smooth 
cutoff function supported in $B_{r}$ such that $\phi \equiv 1$ in $B_{r-\delta / 4}$ where $\delta>0$ is some small positive number which shall be determined later. We write $w^{h}=w_{1}^{h}+w_{2}^{h}$ where $w_{1}^{h}=\phi w^{h}$ and $w_{2}^{h}=(1-\phi) w^{h}$. Take any $x \in B_{r-\delta / 2}$ and $|h|<\delta / 16$. Then $(1-\phi(x)) u(x)=(1-\phi(x)) \tau_{h} u(x)=0$ and $w^{h}(x)=w_{1}^{h}(x)$. We prove that $w_{1}^{h} \in \mathrm{C}^{\alpha+\beta}\left(B_{r-\delta}\right)$ for some $\alpha>0$ with $\alpha+\beta>1$. We note that

$$
\begin{aligned}
& \mathcal{M}_{\mathfrak{Q}_{0}}^{+} w_{1}^{h} \geq \mathcal{M}_{\mathfrak{I}_{0}^{1}}^{+} w_{1}^{h}=\mathcal{M}_{\mathfrak{Q}_{0}^{1}}^{+}\left[w^{h}-w_{2}^{h}\right] \geq 0-\mathcal{M}_{\mathfrak{I}_{0}^{1}}^{+} w_{2^{h}}^{h} \\
& \mathcal{M}_{\mathfrak{I}_{0}}^{-} w_{1}^{h} \leq \mathcal{M}_{\mathfrak{I}_{0}^{1}}^{-} w_{1}^{h}=\mathcal{M}_{\mathfrak{I}_{0}^{1}}^{-}\left[w^{h}-w_{2}^{h}\right] \leq 0-\mathcal{M}_{\mathfrak{I}_{0}^{1}}^{-} w_{2}^{h} .
\end{aligned}
$$

In order to apply Theorem 5.2.2, we have only to show that $\left|\mathcal{M}_{\mathcal{O}_{0}^{1}}^{+} w_{2}^{h}\right|$ and $\left|\mathcal{M}_{\mathcal{Q}_{0}^{1}}^{-} w_{2}^{h}\right|$ are bounded on $B_{r-\delta / 2}$ by $C\|u\|_{L^{\infty}\left(\mathbb{R}^{n}\right)}$ for some universal constant. To show this, we prove that it is true for any operator $\mathcal{L}^{t} \in \mathfrak{L}_{0}^{1}$. Take any $\mathcal{L}^{t} \in \mathfrak{R}_{0}^{1}$. Since $w_{2}^{h} \equiv 0$ on $B_{r-\delta / 2}$, the jump part of $\mathcal{L}^{t} w_{2}^{h}$ vanishes on $B_{r-\delta / 2}$. Thus by the change of variable and the mean value theorem we have that

$$
\begin{aligned}
\mathcal{L}^{t} w_{2}^{h}(x)= & \int_{\mathbb{R}^{n}} \frac{\left(1-\tau_{h} \phi(x+y)\right) \tau_{h} u(x+y)-(1-\phi(x+y)) u(x+y)}{|h|^{\beta}} K(y) d y \\
& \quad+\int_{\mathbb{R}^{n}} \frac{\left(\tau_{h} \phi(x+y)-\phi(x+y)\right) \tau_{h} u(x+y)}{|h|^{\beta}} K(y) d y \\
= & \int_{\mathbb{R}^{n}}(1-\phi(x+y)) u(x+y) \frac{K(y-h)-K(y)}{|h|^{\beta}} d y \\
& \quad+\int_{\mathbb{R}^{n}} \frac{\left(\int_{0}^{1}[\nabla \phi(x+y+\tau h) \cdot h] d \tau\right) \tau_{h} u(x+y)}{|h|^{\beta}} K(y) d y \\
\fallingdotseq & \mathcal{L}_{1}^{t} w_{2}^{h}(x)+\mathcal{L}_{2}^{t} w_{2}^{h}(x)
\end{aligned}
$$

for any $x \in B_{r-\delta / 2}$. Set $\varrho_{1}=\delta / 8$. Since $(1-\phi(x+y)) u(x+y)=0$ for any $x \in B_{r-\delta / 2}$ and $|y|<\delta / 8$, by (5.3.1) we obtain that

$$
\left|\mathcal{L}_{1}^{t} w_{2}^{h}(x)\right| \leq|h|^{1-\beta}\|u\|_{L^{\infty}\left(\mathbb{R}^{n}\right)} \int_{y \in B_{\ell_{1}}^{c}} \frac{|K(y)-K(y-h)|}{|h|} d y \leq C\|u\|_{L^{\infty}\left(\mathbb{R}^{n}\right)}
$$

for any $x \in B_{r-\delta / 2}$ and $|h|<\delta / 16$. Since $\tau_{h} \phi(x+y)=\phi(x+y)$ for every $x \in B_{r-\delta / 2},|y|<\delta / 8$ and $|h|<\delta / 16$, we have that

$$
\left|\mathcal{L}_{2}^{t} w w_{2}^{h}(x)\right| \leq|h|^{1-\beta}|| \nabla \phi\left\|_{L^{\infty}\left(\mathbb{R}^{n}\right)}\right\| u\left\|_{L^{\infty}\left(\mathbb{R}^{n}\right)} \int_{\mathbb{R}^{n} \backslash B_{\varrho_{1}}} \frac{1}{|y|^{n+\sigma}} d y \leq C\right\| u \|_{L^{\infty}\left(\mathbb{R}^{n}\right)}
$$

for any $x \in B_{r-\delta / 2}$ and $|h|<\delta / 16$. Then it follows from (5.3.2), (5.3.3) and (5.3.4) that $\mathcal{M}_{\mathfrak{I}_{0}^{1}}^{+} w_{1}^{h} \geq-C\|u\|_{L^{\infty}\left(\mathbb{R}^{n}\right)}$ and $\mathcal{M}_{\mathfrak{R}_{0}^{1}}^{-} w_{1}^{h} \leq C\|u\|_{L^{\infty}\left(\mathbb{R}^{n}\right)}$ on $B_{r-\delta / 2}$ for any $h \in B_{\delta / 16}$. Thus by Theorem 5.2.2 we have that

$$
\begin{aligned}
\left\|w^{h}\right\|_{C^{\alpha}\left(B_{r-\delta}\right)} & =\left\|w_{1}^{h}\right\|_{C^{\alpha}\left(B_{r-\delta}\right)} \leq C\left\|w_{1}^{h}\right\|_{L^{\infty}\left(B_{r-\delta / 2}\right)}+C\|u\|_{L^{\infty}\left(\mathbb{R}^{n}\right)} \\
& \leq C\|u\|_{C^{0, \beta}\left(B_{r-\delta / 4}\right)}+C\|u\|_{L^{\infty}\left(\mathbb{R}^{n}\right)}
\end{aligned}
$$


for any $h \in B_{\delta / 16}$. By the standard telescopic sum argument [CC], we obtain that $\|u\|_{C^{\alpha+\beta}\left(B_{r-\delta}\right)} \leq C\left(\|u\|_{L^{\infty}\left(\mathbb{R}^{n}\right)}+\left|\mathcal{I}^{ \pm} 0\right|\right)$. Repeating the above argument, after $[1 / \alpha]$-steps we have that

$$
\|u\|_{C^{0,1}\left(B_{3 / 4}\right)} \leq C\left(\|u\|_{L^{\infty}\left(\mathbb{R}^{n}\right)}+\left|\mathcal{I}^{ \pm} 0\right|\right) .
$$

For any unit vector $e \in S^{n-1}$, we consider the following incremental quotients with the same reasoning

$$
w_{e}^{t}(x)=\frac{\tau_{t e} u(x)-u(x)}{h}, t>0 .
$$

If we choose $r=5 / 6, \delta=1 / 3$ and $\beta=1$ in (5.3.5), then by (5.3.5) and (5.3.6) we obtain that $\left\|w_{e}^{t}\right\|_{C^{\alpha}\left(B_{1 / 2}\right)} \leq C\left(\|u\|_{L^{\infty}\left(\mathbb{R}^{n}\right)}+\left|\mathcal{I}^{ \pm} 0\right|\right)$ for any unit vector $e \in S^{n-1}$ and for any $t$ with $|t|<1 / 48$. From this, taking $t \downarrow 0$ we conclude that $\|u\|_{C^{1, \alpha}\left(B_{1 / 2}\right)} \leq C\left(\|u\|_{L^{\infty}\left(\mathbb{R}^{n}\right)}+\left|\mathcal{I}^{ \pm} 0\right|\right)$. Hence we complete the proof.

Acknowledgement. This work had been started during Yong-Cheol Kim was visiting to University of Texas at Austin in the fall semester 2008 for his sabbatical year. He would like to thank for kind hospitality and deep concern of Professor Luis A. Caffarelli during that time. Ki-Ahm Lee was supported by the Korea Research Foundation Grant funded by the Korean Government(MOEHRD, Basic Research Promotion Fund)( KRF-2008-314C00023).

\section{REFERENCES}

[A] Sayah Awatif, Équations d'Hamilton-Jacobi du premier ordre avec termes intégro-différentiels. I Unicité des solutions de viscosité, Comm. Partial Differential Equations 16(6-7), 1991, 1057-1074.

[Ar] M. Arisawa, A new definition of viscosity solutions for a class of second-order degenerate el liptic integro-differential equations, Ann. Inst. H. Poincare Anal. Non Lineaire, 23 (2006), 695-711.

[AT] O. Alvarez and A. Tourin, Viscosity solutions of nonlinear integro-differential equations, Ann. Inst. H. Poincare Anal. Non Lineaire, 13 (1996), 293-317.

[BCI1] G. Barles, E. Chasseigne, and C. Imbert, Hölder continuity of solutions of second-order non-linear elliptic integro-differential equations, 2007. Preprint hal-00179690.

[BCI2] G. Barles, E. Chasseigne, and C. Imbert, On the Dirichlet prob-lem for second-order elliptic integro-differential equations, Indiana Univ. Math. J., 57 (2008), 213-246.

[BI] Guy Barles and Cyril Imbert, Second-order elliptic integro-differential equations: Viscosity solutions' theory revisited, Preprint.

[BBC] Martin T. Barlow; Richard F. Bass; Zhen-Qing Chen; Moritz Kassmann, Non-local Dirichlet forms and symmetric jump processes, Trans. Amer. Math. Soc. 361, 2009, no. 4, 1963-1999.

[BK1] Richard F. Bass and Moritz Kassmann, Harnack inequalities for non-local operators of variable order, Trans. Amer. Math. Soc. 357(2), 2005, 837-850.

[BK2] Richard F. Bass and Moritz Kassmann, Hölder continuity of harmonic functions with respect to operators of variable order, Comm. Partial Differential Equations 30(7-9), 2005, 1249-1259.

[BL] Richard F. Bass and David A. Levin, Harnack inequalities for jump processes, Potential Anal. 17(4), 2002, 375-388.

[BS] I. Bensaoud and A. Sayah, Stability results for Hamilton-Jacobi equations with integrodifferential terms and discontinuous Hamiltonians, Arch. Math. (Basel), 79 (2002), 392-395. 
[C] Luis A. Caffarelli, Interior a priori estimates for solutions of fully nonlinear equations, Ann. of Math. (2) 130(1), 1989, 189-213.

[CS] Luis A. Caffarelli and Luis Silvestre, Regularity theory for fully nonlinear integrodifferential equations, Comm. Pure Appl. Math. 62(5), 2009, 597-638.

[CC] Luis A. Caffarelli and Xavier Cabré, Fully nonlinear elliptic equations, volume 43 of American Mathematical Society, Colloquium Publications. American Mathematical Society, Providence, RI, 1995.

[I] C. Imbert, A non-local regularization of first order Hamilton-Jacobi equations, J. Differential Equations, 211 (2005), 218-246.

[GT] David Gilbarg and Neil S. Trudinger, Elliptic partial differential equations of second order, Second edition. Grundlehren der Mathematischen Wissenschaften [Fundamental Principles of Mathematical Sciences], 224. Springer-Verlag, Berlin, 1983. xiii+513 pp.

[GD] E. De Giorgi and G. Dal Maso, $\Gamma$-convergence and calculus of variations, Lecture notes in Mathematics 979, 1983, Springer-Verlag.

[I] Hitoshi Ishii, On uniqueness and existence of viscosity solutions of fully nonlinear secondorder elliptic PDEs, Comm. Pure Appl. Math. 42(1), 1989, 15-45.

[J] Robert Jensen, The maximum principle for viscosity solutions of fully nonlinear second order partial differential equations, Arch. Rational Mech. Anal. 101(1), 1988, 1-27.

[JK] E. R. Jakobsen and K. H. Karlsen, A Maximum principle for semicontinuous functions applicable to integro-partial differential equations, NoDEA Nonlinear Differential Equations Appl., 13 (2006), 137-165.

[K] M. Kassmann, A priori estimates for integro-differential operators with measurable kernels, Calc. Var. Partial Differential Equations, 34 (2009), pp. 121.

[KL] Yong-Cheol Kim and Ki-Ahm Lee, Regularity results for fully nonlinear integro-differential operators with nonsymmetric positive kernels; Subcritical and Supercritical Cases, in preparation.

[KS] N. V. Krylov and M. V. Safonov, An estimate for the probability of a diffusion process hitting a set of positive measure, Dokl. Akad. Nauk. SSSR 245, 1979, 18-20.

[P] H. Pham, Optimal stopping of control led jump diffusion processes: a viscosity solution approach, J. Math. Systems Estim. Control, 8 (1998), p.27 pp. (electronic).

[S] Luis Silvestre, Hölder estimates for solutions of integro-differential equations like the fractional laplace, Indiana Univ. Math. Jour. 55(3), 2006, 1155-1174.

[So] Halil Mete Soner, Optimal control with state-space constraint. II, SIAM J. Control Optim. 24(6), 1986, 1110-1122.

[SV] Renming Song and Zoran Vondraček, Harnack inequality for some classes of Markov processes, Math. Z. 246(1-2), 2004, 177-202.

- Yong-Cheol Kim : Department of Mathematics Education, Korea University, Seoul 136-701, KoreA

E-mail address: ychkim@korea.ac.kr

- Ki-Ahm Lee : Department of Mathematics, Seoul National University, Seoul 151747, Korea

E-mail address: kiahm@math.snu.ac.kr 